Article

\title{
Extended Thermodynamics for Dense Gases up to Whatever Order and with Only Some Symmetries
}

\author{
Maria Cristina Carrisi ${ }^{1}$, Rita Enoh Tchame ${ }^{2}$, Marcel Obounou ${ }^{2}$ and Sebastiano Pennisi ${ }^{1, *}$ \\ ${ }^{1}$ Dipartimento di Matematica ed Informatica, Università di Cagliari, Via Ospedale 72, \\ 09124 Cagliari, Italy; E-Mail: cristina.carrisi@tiscali.it \\ ${ }^{2}$ Department of Physics, University of Yaoundé I, PO Box 812 Yaoundé, Cameroon; \\ E-Mails: rita.enoh@yahoo.com (R.E.T.); marcelobounou@yahoo.fr (M.O.) \\ * Author to whom correspondence should be addressed; E-Mail: spennisi @ unica.it; \\ Tel.: +39-70-6758533.
}

Academic Editor: Kevin Knuth

Received: 21 August 2015 / Accepted: 13 October 2015 / Published: 16 October 2015

\begin{abstract}
Extended Thermodynamics of dense gases is characterized by two hierarchies of field equations, which allow one to overcome some restrictions on the generality of the previous models. This idea has been introduced by Arima, Taniguchi, Ruggeri and Sugiyama. In the case of a 14-moment model, they have found the closure of the balance equations up to second order with respect to equilibrium. Here, the closure is obtained up to whatever order and imposing only the necessary symmetry conditions. It comes out that the first non-symmetric parts of the higher order fluxes appear only at third order with respect to equilibrium, even if Arima, Taniguchi, Ruggeri and Sugiyama found a non-symmetric part proportional to an arbitrary constant also at first order with respect to equilibrium. Consequently, this constant must be zero, as Arima, Taniguchi, Ruggeri and Sugiyama assumed in the applications and on an intuitive ground.
\end{abstract}

Keywords: extended thermodynamics; dense gas; moments equations 


\section{Introduction}

One of the challenging tasks of modern research is that of modeling non-equilibrium phenomena in which steep gradients and rapid changes occur. Two complementary approaches have been followed in this regard, the kinetic approach and the continuum approach. Here, we follow this second approach, in particular by using the extended thermodynamics (ET) framework, which has many undisputed physical and mathematical advantages.

The first ideas in this regard were proposed by Müller [1] and were based on the modification of the Gibbs relation. Following Ruggeri's criticism [2], a new version was proposed by Liu and Müller [3] and, subsequently, for the relativistic case, by Liu, Müller and Ruggeri [4]; more recent papers in this framework are [5-23].

However, the internal structure of ET implies so strong restrictions to allow only particular state functions; for example, the function $p=p(\rho, T)$ relating the pressure $p$ with the mass density $\rho$ and the absolute temperature $T$ was determined, except for a single-variable function, so that it was adept at describing only particular gases or a continuum (this situation is indicated in Equation (7.9) of [3], for example).

A new approach has been proposed in [24] and other articles, such as [25-38], which overcomes this problem by considering two blocks of balance equations. In this framework, we have recently studied two different cases: one deals with rarefied polyatomic gases [37], even if the reduced set of applicability is not indicated in the title of that paper. The other one is presented here and deals with dense gases. These two cases are like two branches of the same tree; consequently, the description of their common trunk is the same, and we report it here for the sake of completeness, but with the prospect of the new application. We will address below the new points of the present article, for example after Equation (5) and four lines after Equation (9). The results will be expressed in terms of a function $H$, which will result in the sum of the corresponding expression in [37] and of a new incremental term; the present study aims to find this new term.

Therefore, let us begin with the description of the above-mentioned common trunk; in particular, in the 14-moment case treated in [24], the two blocks of balance equations are:

$$
\begin{aligned}
& \partial_{t} F+\partial_{k} F^{k}=0, \quad \partial_{t} F^{i}+\partial_{k} F^{k i}=0, \quad \partial_{t} F^{i j}+\partial_{k} F^{k i j}=P^{i j}, \\
& \partial_{t} G+\partial_{k} G^{k}=0, \quad \partial_{t} G^{i}+\partial_{k} G^{k i}=Q^{i},
\end{aligned}
$$

here $(1)_{1,2}$ are the conservation laws of mass and momentum, while $(1)_{4}$ is the conservation laws of energy. The block $(1)_{1-3}$ is called the "mass block" while $(1)_{4,5}$ is called the "energy block".

Equation (1) can be written in a more compact form as:

$$
\begin{gathered}
\partial_{t} F^{A}+\partial_{k} F^{k A}=P^{A}, \\
\text { where } \quad F^{A}=\left(F^{N}, G^{E}\right), \quad F^{k A}=\left(F^{k N}, G^{k E}\right), \quad P^{A}=\left(P^{N}, Q^{E}\right) .
\end{gathered}
$$

In Equation (2), the constitutive functions $F^{k i j}, G^{k i}, P^{i j}, Q^{i}$ appear. Restrictions on their generalities are obtained by imposing the entropy principle, the symmetry conditions and the Galilean relativity principle. 
The first of these principles can be exploited through Liu's theorem [39] and by using a bright idea conceived of by Ruggeri [40]; so, it becomes equivalent to assuming the existence of Lagrange multipliers $\mu_{A}$, which can be taken as independent variables, and after that, we have:

$$
F^{A}=\frac{\partial h^{\prime}}{\partial \mu_{A}}, \quad F^{k A}=\frac{\partial h^{\prime k}}{\partial \mu_{A}},
$$

which expresses all of the moments in terms of only two unknown functions, the four-potentials $h^{\prime}, h^{\prime k}$. A consequence of Equation (3) is that the field equations assume the symmetric form, thus assuring hyperbolicity if $h^{\prime}$ is a convex function of its variables.

Other restrictions are given by the symmetry conditions, that is the flux in each balance equation is equal to the independent variable in the subsequent equation, except for the flux in the last equation of the mass block and of that in the energy block. Moreover, $F^{i j}$ is a symmetric tensor.

Thanks to Equation (3), these conditions assume the form:

$$
\frac{\partial h^{\prime}}{\partial \mu_{i}}=\frac{\partial h^{\prime i}}{\partial \mu}, \quad \frac{\partial h^{\prime}}{\partial \mu_{i j}}=\frac{\partial h^{i}}{\partial \mu_{j}}, \quad \frac{\partial h^{\prime}}{\partial \lambda_{i}}=\frac{\partial h^{\prime i}}{\partial \lambda},
$$

(where we have assumed the decomposition $\mu_{A}=\left(\mu, \mu_{i}, \mu_{i j}, u p \lambda, \lambda_{i}\right)$ for the Lagrange multipliers) and $\mu_{i j}$ is a symmetric tensor.

Eventual supplementary symmetry conditions are those imposing the symmetry of the tensors $F^{k i j}$ and $G^{k i}$ and are motivated by the kinetic counterpart of this theory. Thanks to Equation (3), these conditions may be expressed as:

$$
\frac{\partial h^{\prime[k}}{\partial \mu_{i] j}}=0, \frac{\partial h^{\prime[k}}{\partial \lambda_{i]}}=0 .
$$

These supplementary symmetry conditions were not imposed in [24] because in that article, the phenomenological approach was adopted in order to construct the extended thermodynamics of dense gases. After the development of this theory, the kinetic theoretical approach was proposed in [28], but only for rarefied polyatomic gases, not dense gases. For this reason, in the balance Equation (1), the moments appear as a distribution function, which is generalized, so that it depends also on an internal energy parameter $I$; as a consequence, the supplementary symmetry condition Equation (5) has to be imposed for this case.

In [37], the general solution has been found up to whatever order with respect to the equilibrium of the condition Equations (4) and (5) and of the below reported Equation (6). Therefore, the general solution for the rarefied polyatomic gases has been completely exploited.

In the present article, we aim to obtain the general solution without imposing the supplementary condition Equation (5) in order to have the model for dense gases and in agreement with the article [24].

The next conditions come from the Galilean relativity principle. A natural way to impose this principle is described in [33] for the 18-moment model. It extends, to the two-block theory, the method described in [41] with the further deepening of [42] for the old one-block theory. The resulting 
Equations (13) and (14) of [33] contain two additional variables with respect to the present model, that is $\mu_{i l l}$ and $\lambda_{l l}$; by making these variables equal to zero, we obtain the counterpart for our model, that is:

$$
\begin{aligned}
& \frac{\partial h^{\prime}}{\partial \mu} \mu_{i}+\frac{\partial h^{\prime}}{\partial \mu_{h}}\left(2 \mu_{i h}+2 \lambda \delta_{h i}\right)+2 \frac{\partial h^{\prime}}{\partial \mu_{h i}} \lambda_{h}+\frac{\partial h^{\prime}}{\partial \lambda} \lambda_{i}=0, \\
& \frac{\partial h^{\prime k}}{\partial \mu} \mu_{i}+\frac{\partial h^{\prime k}}{\partial \mu_{h}}\left(2 \mu_{i h}+2 \lambda \delta_{h i}\right)+2 \frac{\partial h^{\prime k}}{\partial \mu_{h i}} \lambda_{h}+\frac{\partial h^{\prime k}}{\partial \lambda} \lambda_{i}+h^{\prime} \delta^{k i}=0 .
\end{aligned}
$$

Now, by using Equation (4), we note that the derivative of $(6)_{1}$ with respect to $\mu_{k}$ is equal to the derivative of $(6)_{2}$ with respect to $\mu$; similarly, the derivative of $(6)_{1}$ with respect to $\lambda_{k}$ is equal to the derivative of $(6)_{2}$ with respect to $\lambda$.

Consequently, the left-hand side of Equation $(6)_{1}$ is a vectorial function depending only on two scalars $\mu, \lambda$ and on a symmetric tensor $\mu_{i j}$. For the representation theorems $[43,44]$, it can be only zero, and for this reason, we do not need to impose $(6)_{1}$.

This result, combined with the above conditions coming from the entropy principle and the symmetry conditions, will be that a scalar function $H$ exists, such that:

$$
\begin{gathered}
h^{\prime}=\frac{\partial H}{\partial \mu}, \quad h^{\prime i}=\frac{\partial H}{\partial \mu_{i}} . \\
\frac{\partial^{2} H}{\partial \mu \partial \mu_{i j}}=\frac{\partial^{2} H}{\partial \mu_{i} \partial \mu_{j}}, \quad \frac{\partial^{2} H}{\partial \mu \partial \lambda_{i}}=\frac{\partial^{2} H}{\partial \lambda \partial \mu_{i}} . \\
\frac{\partial^{2} H}{\partial \mu \partial \mu_{k}} \mu_{i}+2 \frac{\partial^{2} H}{\partial \mu \partial \mu_{k j}} \mu_{j i}+2 \frac{\partial^{2} H}{\partial \mu \partial \mu_{k i}} \lambda+2 \frac{\partial^{2} H}{\partial \mu_{k} \partial \mu_{i j}} \lambda_{j}+\frac{\partial^{2} H}{\partial \mu \partial \lambda_{k}} \lambda_{i}+\frac{\partial H}{\partial \mu} \delta^{k i}=0 .
\end{gathered}
$$

The challenge is now to find the general solution of our conditions, up to whatever order with respect to equilibrium. This is defined as the state where $\mu_{i}=0, \mu_{i j}=0, \lambda_{i}=0$, so that the only non-zero variables are $\mu$ and $\lambda$.

Now, in [37], the general solution has been found, up to whatever order with respect to equilibrium, of the condition Equations (8) and (9) and also of Equation (5); but, for a closer agreement with the article [24], we want now to do this without imposing Equation (5).

However, although it may seem strange, with less conditions, the calculations become heavier! In fact, if it was possible to use the condition Equation (5), then the function $H$ can be expressed as a sum of a function, which has derivatives that are symmetric tensors (so that we can also easily write its expansion), and of a function depending only on $\mu_{a b}, \lambda, \lambda_{c}$ (so that it does not contribute to Equations (5), (8) and (9).

Now, in the present article, we cannot use this property, because we do not have the constraint Equation (5). To overcome this difficulty, we proceed as follows. Firstly,

1. We show a particular solution of Equations (8) and (9).

It is $H=H_{1}$, where:

$$
\begin{array}{r}
H_{1}=\sum_{p, q}^{0 \cdots \infty} \sum_{r \in I_{p}} \frac{1}{p !} \frac{1}{q !} \frac{1}{r !} \frac{(p+2 q+r+1) ! !}{p+2 q+r+1} \frac{\partial^{r+p}}{\partial \lambda^{r} \partial \mu^{p}}\left[\left(\frac{-1}{2 \lambda}\right)^{q+\frac{p+r}{2}} \psi_{\frac{p+r}{2}}\right] . \\
\cdot \delta^{\left(i_{1} \cdots i_{p} h_{1} k_{1} \cdots h_{q} k_{q} j_{1} \cdots j_{r}\right)} \mu_{i_{1}} \cdots \mu_{i_{p}} \mu_{h_{1} k_{1}} \cdots \mu_{h_{q} k_{q}} \lambda_{j_{1}} \cdots \lambda_{j_{r}}
\end{array}
$$


and $\psi_{n}(\mu, \lambda)$ is a family of functions constrained only by:

$$
\frac{\partial}{\partial \mu} \psi_{n+1}=\psi_{n} \quad \text { for } \quad n \geq 0
$$

In Equation (10), the symbol $r \in I_{p}$ means that the summation is limited to the values of $r$, such that $r+p$ is even.

In Appendix 1, the proof is reported for the fact that $H=H_{1}$ is a solution of Equations (8) and (9). Moreover, $\left(H_{1}\right)_{e q .}=\psi_{0}(\mu, \lambda)$, which is an arbitrary two-variable function, such as $H_{e q}$.

So we can identify $\quad \psi_{0}(\mu, \lambda)=H_{e q}$ and define $\quad \Delta H=H-H_{1}$.

In this way, the condition Equations (8) and (9) become:

$$
\begin{gathered}
\frac{\partial^{2} \Delta H}{\partial \mu \partial \mu_{i j}}=\frac{\partial^{2} \Delta H}{\partial \mu_{i} \partial \mu_{j}}, \quad \frac{\partial^{2} \Delta H}{\partial \mu \partial \lambda_{i}}=\frac{\partial^{2} \Delta H}{\partial \lambda \partial \mu_{i}} \\
\frac{\partial^{2} \Delta H}{\partial \mu \partial \mu_{k}} \mu_{i}+2 \frac{\partial^{2} \Delta H}{\partial \mu \partial \mu_{k j}} \mu_{j i}+2 \frac{\partial^{2} \Delta H}{\partial \mu \partial \mu_{k i}} \lambda+2 \frac{\partial^{2} \Delta H}{\partial \mu_{k} \partial \mu_{i j}} \lambda_{j}+\frac{\partial^{2} \Delta H}{\partial \mu \partial \lambda_{k}} \lambda_{i}+\frac{\partial \Delta H}{\partial \mu} \delta^{k i}=0 \\
\text { and we have also } \quad(\Delta H)_{e q .}=0 .
\end{gathered}
$$

Now, an interesting consequence of Equations (13) and (14) is that:

Property 1: " The expansion of $\Delta H$ up to order $n \geq 1$ with respect to equilibrium is a polynomial of degree $n-1$ in the variable $\mu$."

We report in Appendix 2 the proof of the property.

Now, a well-known elementary mathematical property is that the Taylor expansion of a polynomial is not an approximate expression of that polynomial function, but is exactly equal to it. We can also assume an expansion of infinity order for that polynomial, with zero coefficients for the terms of order greater than the degree of the polynomial itself.

Moreover, we have deduced this property from Equations (13) and (14); consequently, it will be not necessary to take into account what coefficients are zero, since on the resulting expansion, we will impose again Equations (13) and (14).

Thanks to these properties, it is not restrictive to assume for $\Delta H$ a polynomial expansion of infinity order in the variable $\mu$; this fact allows us to treat the variable $\mu$ as the other Lagrange multipliers $\mu_{i}$ and $\mu_{i j}$ of the block $(1)_{1}$, because we had already a polynomial expansion for them.

Therefore, even if $\mu$ is not zero at equilibrium, as concerns $\Delta H$, we can do an expansion also around $\mu=0$; obviously, the situation is different for the particular solution $H_{1}$ reported in Equation (10). Therefore, the physical meaning of a non-polynomial expression in the variable $\mu$ remains charged only to the solution $H_{1}$.

The next step with which we proceed is the following one:

2. We note that $\frac{\partial^{2} \Delta H}{\partial \mu^{2}}$ has symmetric tensors as derivatives.

The details in this regard are reported in Section 2, and also, they will imply the following expression for $\frac{\partial \Delta H}{\partial \mu}$ : 


$$
\begin{gathered}
\frac{\partial \Delta H}{\partial \mu}=\sum_{p, q, s}^{0 \cdots \infty} \sum_{r \in I_{p}} \frac{1}{p !} \frac{1}{q !} \frac{1}{r !} \frac{1}{s !} \vartheta_{p, q, r, s}(\lambda) \mu^{s} \delta^{\left(i_{1} \cdots i_{p} h_{1} k_{1} \cdots h_{q} k_{q} j_{1} \cdots j_{r}\right)} \\
\mu_{i_{1}} \cdots \mu_{i_{p}} \mu_{h_{1} k_{1}} \cdots \mu_{h_{q} k_{q}} \lambda_{j_{1}} \cdots \lambda_{j_{r}}++H^{* 0}\left(\mu_{a b}, \lambda, u p \lambda_{c}\right) .
\end{gathered}
$$

with:

$$
\vartheta_{0, q, r, 0}(\lambda)=0, \quad \vartheta_{0,0,0, s}(\lambda)=0 \quad \text { for } \quad s \geq 0, \quad H^{* 0}\left(0_{a b}, \lambda, 0_{c}\right)=0 .
$$

Further restrictions are consequences of Equation (13) and will be considered in Subsection 2.1. They are expressed by Equation (26) and will be useful for the sequel.

In Section 3, restrictions will be found for the scalar functions appearing in Equation (15), by analyzing Equation (26) and the derivative of Equation (13) with respect to $\mu$. In Section 4, Equation (15) will be integrated, and arbitrary functions will arise from integration; moreover, the condition Equation (13) (not derivated) will restrict their generality. The solution of these restrictions will be found in Sections 5 and 6. Finally, conclusions will be drawn.

It is not difficult to report in explicit form the fields equations up to whatever order; but a very long expression is not elegant and may give to this article the aspect of a mere database. Therefore, we prefer to show how to write them by simple taking some derivatives without repeating the present calculations. To this end, let us consider firstly $H=H_{1}+\Delta H$ with $H_{1}$ given by Equation (10) and $\Delta H$ given by the below Equation (54); then, let us write $h^{\prime}$ and $h^{\prime k}$ from Equation (7).

After that, let us substitute them into Equation (3), which can be written in explicit form as:

$$
\begin{aligned}
& F=\frac{\partial h^{\prime}}{\partial \mu}, F^{i}=\frac{\partial h^{\prime}}{\partial \mu_{i}}, F^{i j}=\frac{\partial h^{\prime}}{\partial \mu_{i j}}, G^{l l}=\frac{\partial h^{\prime}}{\partial \lambda}, G^{i l l}=\frac{\partial h^{\prime}}{\partial \lambda_{i}}, \\
& F^{k i j}=\frac{\partial h^{\prime k}}{\partial \mu_{i j}}, G^{k i l l}=\frac{\partial h^{\prime k}}{\partial \lambda_{i}} .
\end{aligned}
$$

By substituting these expressions in Equation (1), we obtain partial differential equations for the determination of the unknown functions $\mu_{A}(\vec{x}, t)$ and $\lambda_{A}(\vec{x}, t)$. Finally, by substituting these last functions into Equation (17), we obtain how the fields evolve in space and time. Someone may object that the Lagrange multipliers have no physical meaning; this is true, but at the end, we obtain in any case the fields $F^{A}$ and $F^{k A}$. The situation has a counterpart in the geometry of a surface. This can be given through parametric equations, and the parameters have no geometrical meaning, however, nobody objects for this reason to use parametrical equations of a surface.

Obviously, from this situation, it is evident that the Lagrange multipliers are special parameters, and in fact, for this reason, they have been called "main field" in [40].

Alternatively, $(17)_{1-5}$ may be used to obtain the Lagrange multipliers in terms of $F, F^{i}, F^{i j}, G^{l l}, G^{\text {ill }}$ and then to substitute them into $(17)_{6-7}$; the invertibility is surely possible, but the resulting expressions are very long and complicated, so that only with a computer program this is possible, after having chosen the order in which to stop the process.

A more fine procedure is to follow the same iter, but with the Galilean invariant parts of the fields. In this case, instead of Equation (17), we have to consider the relations:

$$
\rho=\frac{\partial h^{\prime}}{\partial \mu}, 0=\frac{\partial h^{\prime}}{\partial \mu_{i}}, M^{i j}=\frac{\partial h^{\prime}}{\partial \mu_{i j}}, m^{i i}=\frac{\partial h^{\prime}}{\partial \lambda}, m^{i p p}=\frac{\partial h^{\prime}}{\partial \lambda_{i}}
$$


to obtain the velocity-independent parts of the Lagrange multipliers in terms of $M^{i j}, m^{i i}, m^{i p p}$ and of the mass density $\rho$; after that, we have to substitute them into:

$$
M^{k i j}=\frac{\partial h^{\prime k}}{\partial \mu_{i j}}, m^{k i p p}=\frac{\partial h^{\prime k}}{\partial \lambda_{i}}
$$

and, finally, in Equation (8) of [24]. Furthermore, in this case, the resulting expressions are very long and complicated and may be written only after having chosen the order in which to stop the process.

We conclude this section noting that, since the non-symmetric part of the higher order fluxes appear only at third order with respect to equilibrium, we have that the closure up to second order satisfies all of the symmetry conditions (also the supplementary ones), so that it is equal to that of [37]. In that paper, it has been proven also that its results reproduce exactly those of [24] in the approximation near the equilibrium state, provided that an arbitrary constant is considered zero, as also the authors of [24] have done in all of the applications and on an intuitive ground. Consequently, the same thing can be said for the present results; this gives a strong confirmation of the results in [24], because it furnishes proof that a higher order approach does not limit also the results near equilibrium. Obviously, the article [24] did not need this confirmation, but it will not do any harm.

For the sake of completeness, we report here the constitutive equations with the second order closure with respect to equilibrium, which have not been achieved in [24].

Let us begin by writing the field equations in the physical variables $\rho, T, v_{i}, \pi, S^{<i j>}, q^{i}$, as can be found from Equation (9) of [24] by taking into account Equations (10)-(12) of the same article. They are:

$$
\begin{gathered}
\frac{d}{d t} \rho+\rho \frac{\partial v_{k}}{\partial x_{k}}=0, \quad \rho \frac{d}{d t} v_{i}+\frac{\partial\left[(p+\pi) \delta^{i j}-S^{<i j>}\right]}{\partial x_{j}}=0 \\
\frac{d}{d t}(2 \rho \epsilon)+2 \rho \epsilon \frac{\partial v_{k}}{\partial x_{k}}+2 \frac{\partial q_{k}}{\partial x_{k}}+2\left[(p+\pi) \delta^{i k}-S^{<i k>}\right] \frac{\partial v_{i}}{\partial x_{k}}=0 \\
\frac{d}{d t}(p+\pi)+(p+\pi) \frac{\partial v_{k}}{\partial x_{k}}+\frac{1}{3} \frac{\partial M_{i i k}}{\partial x_{k}}+\frac{2}{3}\left[(p+\pi) \delta^{i k}-S^{<i k>}\right] \frac{\partial v_{i}}{\partial x_{k}}=P_{i i} \\
\frac{d}{d t} S^{<i j>}+S^{<i j>} \frac{\partial v_{k}}{\partial x_{k}}-\frac{\partial M_{<i j>k}}{\partial x_{k}}-2(p+\pi) \frac{\partial v_{<i}}{\partial x_{j>}} \\
+\left(S^{<k j>} \frac{\partial v_{i}}{\partial x_{k}}+S^{<k i>} \frac{\partial v_{j}}{\partial x_{k}}-\frac{2}{3} S^{<k a>} \frac{\partial v_{a}}{\partial x_{k}} \delta^{i j}\right)=-P_{<i j>} \\
\frac{d}{d t} q^{i}+q^{i} \frac{\partial v_{k}}{\partial x_{k}}+\frac{1}{2} \frac{\partial m_{p p i k}}{\partial x_{k}}+M_{p i k} \frac{\partial v_{p}}{\partial x_{k}}+q^{k} \frac{\partial v_{i}}{\partial x_{k}} \\
+\left[(p+\pi) \delta^{i p}-S^{<i p>}\right] \frac{d}{d t} v_{p}+\rho \epsilon \frac{d}{d t} v_{i}=\frac{1}{2} Q_{i}
\end{gathered}
$$

where $\frac{d}{d t}$ denotes the Lagrangian derivative with respect to time.

The constitutive functions appearing in these equations are $M^{i j k}, m^{p p i k}$, besides the production terms about which we add nothing more to what is written in [24]. We find: 


$$
\begin{gathered}
M^{i j k}=\frac{3}{2} K q^{(i} \delta^{j k)}+3 K_{1}\left(S^{(<i j>} q^{k)}+\delta^{(i j} S^{<k) c>} q_{c}\right) \\
+\frac{3}{2} K_{2} \pi \delta^{(i j} q^{k)}-\frac{3}{2} K_{3} \delta^{(i j} S^{<k) c>} q_{c} \\
m^{p p i k}=\beta_{1} \delta^{i k}+\pi \delta^{i k}\left[\frac{5}{12} \frac{h_{4}}{h_{2}} K-\frac{h_{4}}{2 h_{2}} \frac{\frac{\partial P}{\partial T}}{\rho \frac{\partial \epsilon}{\partial T}}+2\left(\epsilon+\frac{p}{\rho}\right)\right]-S^{<i k>}\left[\frac{h_{4}}{2 h_{3}} K+2\left(\epsilon+\frac{p}{\rho}\right)\right] \\
+B_{2} \pi^{2} \delta^{i k}+B_{3} S_{<a b>} S_{<a b>} \delta^{i k}+B_{4} q_{a} q_{a} \delta^{i k}+B_{5} q^{i} q^{k}+B_{6} \pi S^{<i k>} \\
+B_{7} \pi S^{<i a>} S^{<a k>} .
\end{gathered}
$$

The expressions of the coefficients are reported in [24] and Equations (90)-(93) of [37]; we do not copy them here for the sake of brevity.

\section{On the Symmetry of the Derivatives of $\frac{\partial^{2} \Delta H}{\partial \mu^{2}}$ and Its Consequences}

We note that $\frac{\partial^{2} \Delta H}{\partial \mu^{2}}$ has symmetric tensors as derivatives. In fact, from the derivatives of $(13)_{1,2}$ with respect to $\mu_{k}$, we can take the skew-symmetric part with respect to $i$ and $k$, so obtaining:

$$
\frac{\partial^{3} \Delta H}{\partial \mu \partial \mu_{[k} \partial \mu_{i] j}}=0, \quad \frac{\partial^{3} \Delta H}{\partial \mu \partial \mu_{[k} \partial \lambda_{i]}}=0 .
$$

From the second derivatives of $(13)_{1,2}$ with respect to $\mu$ and $\mu_{a b}$, we can take the skew-symmetric part with respect to $i$ and $b$, so obtaining:

$$
\frac{\partial^{4} \Delta H}{\partial \mu^{2} \partial \mu_{a[b} \partial \mu_{i] j}}=\frac{\partial^{4} \Delta H}{\partial \mu \partial \mu_{a[b} \partial \mu_{i]} \partial \mu_{j}}=0, \quad \frac{\partial^{4} \Delta H}{\partial \mu^{2} \partial \mu_{a[b} \partial \lambda_{i]}}=\frac{\partial^{4} \Delta H}{\partial \mu \partial \lambda \partial \mu_{a[b} \partial \mu_{i]}}=0
$$

where Equation (20) has been used in the second passage.

Equation (21) and the derivatives of Equation (20), with respect to $\mu$, prove our property. We now prove that $\frac{\partial \Delta H}{\partial \mu}$ is the sum of $H^{* 0}\left(\mu_{a b}, \lambda, \lambda_{c}\right)$ and of a scalar function whose derivatives are all symmetric tensors.

In fact, from Equation (20), we deduce that $\frac{\partial^{2} \Delta H}{\partial \mu_{k} \partial \mu}$ has all symmetric derivatives, so that its expansion around equilibrium is of the type:

$$
\frac{\partial^{2} \Delta H}{\partial \mu_{k} \partial \mu}=\sum_{p, q}^{0 \cdots \infty} \sum_{r \in I_{p+1}} \frac{1}{p !} \frac{1}{q !} \frac{1}{r !} H_{p, q, r}^{*} \delta^{\left(k i_{1} \cdots i_{p} h_{1} k_{1} \cdots h_{q} k_{q} j_{1} \cdots j_{r}\right)} \mu_{i_{1}} \cdots \mu_{i_{p}} \mu_{h_{1} k_{1}} \cdots \mu_{h_{q} k_{q}} \lambda_{j_{1}} \cdots \lambda_{j_{r}} .
$$

By integrating this expression with respect to $\mu_{k}$, we obtain:

$$
\begin{array}{r}
\frac{\partial \Delta H}{\partial \mu}=\sum_{p, q}^{0 \cdots \infty} \sum_{r \in I_{p+1}} \frac{1}{(p+1) !} \frac{1}{q !} \frac{1}{r !} H_{p, q, r}^{*} \delta^{\left(i_{1} \cdots i_{p+1} h_{1} k_{1} \cdots h_{q} k_{q} j_{1} \cdots j_{r}\right)} \\
\mu_{i_{1}} \cdots \mu_{i_{p+1}} \mu_{h_{1} k_{1}} \cdots \mu_{h_{q} k_{q}} \lambda_{j_{1}} \cdots \lambda_{j_{r}}+H^{*}\left(\mu, \mu_{a b}, \lambda, \lambda_{c}\right) .
\end{array}
$$


The derivative of Equation (22) with respect to $\mu$ is:

$$
\begin{array}{r}
\frac{\partial^{2} \Delta H}{\partial \mu^{2}}=\sum_{p, q}^{0 \cdots \infty} \sum_{r \in I_{p+1}} \frac{1}{(p+1) !} \frac{1}{q !} \frac{1}{r !} \frac{\partial H_{p, q, r}^{*}}{\partial \mu} \delta^{\left(i_{1} \cdots i_{p+1} h_{1} k_{1} \cdots h_{q} k_{q} j_{1} \cdots j_{r}\right)} \\
\mu_{i_{1}} \cdots \mu_{i_{p+1}} \mu_{h_{1} k_{1}} \cdots \mu_{h_{q} k_{q}} \lambda_{j_{1}} \cdots \lambda_{j_{r}}+\frac{\partial H^{*}}{\partial \mu} .
\end{array}
$$

However, also $\frac{\partial^{2} \Delta H}{\partial \mu^{2}}$ has all symmetric derivatives, so that its expansion is:

$$
\begin{array}{r}
\frac{\partial^{2} \Delta H}{\partial \mu^{2}}=\sum_{p, q}^{0 \cdots \infty} \sum_{r \in I_{p}} \frac{1}{p !} \frac{1}{q !} \frac{1}{r !} \frac{\partial H_{p, q, r}(\mu, \lambda)}{\partial \mu} \delta^{\left(i_{1} \cdots i_{p} h_{1} k_{1} \cdots h_{q} k_{q} j_{1} \cdots j_{r}\right)} \\
\mu_{i_{1}} \cdots \mu_{i_{p}} \mu_{h_{1} k_{1}} \cdots \mu_{h_{q} k_{q}} \lambda_{j_{1}} \cdots \lambda_{j_{r}}
\end{array}
$$

where $H_{p, q, r}$ appears through its derivative with respect to $\mu$ for later convenience and without loss of generality.

By substituting Equation (24) into Equation (23), we find an expression from which we deduce $\frac{\partial H^{*}}{\partial \mu}$; by integrating it, we obtain:

$$
\begin{array}{r}
H^{*}=\sum_{p, q}^{0 \cdots \infty} \sum_{r \in I_{p}} \frac{1}{p !} \frac{1}{q !} \frac{1}{r !} H_{p, q, r}(\mu, \lambda) \delta^{\left(i_{1} \cdots i_{p} h_{1} k_{1} \cdots h_{q} k_{q} j_{1} \cdots j_{r}\right)} \mu_{i_{1}} \cdots \mu_{i_{p}} \mu_{h_{1} k_{1}} \cdots \mu_{h_{q} k_{q}} \lambda_{j_{1}} \\
\cdots \lambda_{j_{r}}-\sum_{p, q}^{0 \cdots \infty} \sum_{r \in I_{p+1}} \frac{1}{(p+1) !} \frac{1}{q !} \frac{1}{r !} H_{p, q, r}^{*} \delta^{\left(i_{1} \cdots i_{p+1} h_{1} k_{1} \cdots h_{q} k_{q} j_{1} \cdots j_{r}\right)} \\
\mu_{i_{1}} \cdots \mu_{i_{p+1}} \mu_{h_{1} k_{1}} \cdots \mu_{h_{q} k_{q}} \lambda_{j_{1}} \cdots \lambda_{j_{r}}+H^{* 0}\left(\mu_{a b}, \lambda, \lambda_{c}\right),
\end{array}
$$

where $H^{* 0}$ arises from an integration with respect to $\mu$, so that it does not depend on $\mu$; moreover, it does not depend on $\mu_{i}$, because $H^{*}$ does not depend on $\mu_{i}$.

By substituting this expression into Equation (22), we find that $\frac{\partial \Delta H}{\partial \mu}$ is the sum of $H^{* 0}\left(\mu_{a b}, \lambda, \lambda_{c}\right)$ and of a function whose derivatives are all symmetric tensors; consequently, it can be written in the form:

$$
\begin{array}{r}
\frac{\partial \Delta H}{\partial \mu}=\sum_{p, q}^{0 \cdots \infty} \sum_{r \in I_{p}} \frac{1}{p !} \frac{1}{q !} \frac{1}{r !} H_{p, q, r}(\mu, \lambda) \delta^{\left(i_{1} \cdots i_{p} h_{1} k_{1} \cdots h_{q} k_{q} j_{1} \cdots j_{r}\right)} \\
\mu_{i_{1}} \cdots \mu_{i_{p}} \mu_{h_{1} k_{1}} \cdots \mu_{h_{q} k_{q}} \lambda_{j_{1}} \cdots \lambda_{j_{r}}+H^{* 0}\left(\mu_{a b}, \lambda, \lambda_{c}\right) .
\end{array}
$$

If we take into account the result of Property 1, we see that also $\frac{\partial \Delta H}{\partial \mu}$ can be expressed as a polynomial of infinite degree in $\mu$, so that Equation (25) can be written as Equation (15). Now, if we substitute into Equation (15) $H^{* 0}$ with $H^{* 0 N}-\sum_{q}^{0 \cdots \infty} \sum_{r \in I_{0}} \frac{1}{q !} \frac{1}{r !} \vartheta_{0, q, r, 0}(\lambda) \delta^{\left(h_{1} k_{1} \cdots h_{q} k_{q} j_{1} \cdots j_{r}\right)} \mu_{h_{1} k_{1}} \cdots \mu_{h_{q} k_{q}} \lambda_{j_{1}} \cdots \lambda_{j_{r}}$, we note that Equation (15) remains unchanged, except that now, we have $H^{* 0 N}$ instead of $H^{* 0}$ and zero instead of $\vartheta_{0, q, r, 0}$. We conclude that we may still use Equation (15) and assume, without loss of generality, that $(16)_{1}$ holds.

If we calculate Equation (15) at equilibrium and take into account Equation (14), we obtain: $0=\sum_{s=0}^{\infty} \frac{1}{s !} \vartheta_{0,0,0, s}(\lambda) \mu^{s}+H^{* 0}\left(0_{a b}, \lambda, 0_{c}\right)$.

Consequently, we have $\vartheta_{0,0,0, s}(\lambda)=0$ for $s \geq 1$, and from Equation $(16)_{1}$, it follows $(16)_{2}$. Moreover, Equation (14) will give $(16)_{3}$. 


\section{Further Restrictions}

For the sequel, it will be useful to consider some consequences of Equation (13). They are:

$$
\begin{gathered}
\frac{\partial^{3} \Delta H}{\partial \mu_{j} \partial \lambda_{i} \partial \mu}=\frac{\partial^{3} \Delta H}{\partial \lambda \partial \mu_{i j} \partial \mu} \quad, \\
{\left[\frac{\partial^{2} \Delta H}{\partial \mu \partial \mu_{k}} \mu_{i}+2 \frac{\partial^{2} \Delta H}{\partial \mu \partial \mu_{k j}} \mu_{j i}+2 \frac{\partial^{2} \Delta H}{\partial \mu \partial \mu_{k i}} \lambda+\frac{\partial \Delta H}{\partial \mu} \delta^{k i}\right]_{\lambda_{j}=0}=0} \\
\frac{\partial^{3} \Delta H}{\partial \mu_{a} \partial \mu_{k} \partial \mu} \mu_{i}+2 \frac{\partial^{2} \Delta H}{\partial \mu \partial \mu_{(k}} \delta_{a) i}+2 \frac{\partial^{3} \Delta H}{\partial \mu_{a} \partial \mu_{k j} \partial \mu} \mu_{j i}+2 \frac{\partial^{3} \Delta H}{\partial \mu_{a} \partial \mu_{k i} \partial \mu} \lambda \\
+\frac{\partial^{3} \Delta H}{\partial \mu_{a} \partial \lambda_{k} \partial \mu} \lambda_{i}+2 \frac{\partial^{3} \Delta H}{\partial \mu_{i j} \partial \mu_{k a} \partial \mu} \lambda_{j}=0 \\
\frac{\partial^{3} \Delta H}{\partial \mu_{k i} \partial \mu \partial \lambda} \mu_{i}+2 \frac{\partial^{3} \Delta H}{\partial \mu_{k j} \partial \mu \partial \lambda} \mu_{j i}+2 \frac{\partial^{3} \Delta H}{\partial \mu_{k i} \partial \mu \partial \lambda} \lambda+2 \frac{\partial^{2} \Delta H}{\partial \mu_{k i} \partial \mu} \\
+\frac{\partial^{3} \Delta H}{\partial \lambda_{k} \partial \mu \partial \lambda} \lambda_{i}+\frac{\partial^{2} \Delta H}{\partial \lambda \partial \mu} \delta^{k i}+2 \frac{\partial^{3} \Delta H}{\partial \mu_{i j} \partial \lambda_{k} \partial \mu} \lambda_{j}=0
\end{gathered}
$$

The first one of these equations is obtained by taking the derivatives of $(13)_{2}$ with respect to $\mu_{j}$ and by substituting in its right-hand side $\frac{\partial^{2} \Delta H}{\partial \mu_{i} \partial \mu_{j}}$ from $(13)_{1}$; the second one is obtained by simply calculating $(13)_{3}$ in $\lambda_{j}=0$; similarly, $(26)_{3}$ is obtained by taking the derivative of $(13)_{3}$ with respect to $\mu_{a}$ and, subsequently, by substituting in its fourth term $\frac{\partial^{2} \Delta H}{\partial \mu_{k} \partial \mu_{a}}$ from $(13)_{1}$. Finally, in the derivative of $(13)_{3}$ with respect to $\lambda$, we can substitute $\frac{\partial^{2} \Delta H}{\partial \lambda \partial \mu_{k}}$ from $(13)_{2}$ in its fourth term; in this way, $(26)_{4}$ is obtained.

We see that Equation (26) is the conditions on $\frac{\partial \Delta H}{\partial \mu}$, so that they may be considered a sort of integrability condition on $\Delta H$, if $\frac{\partial \Delta H}{\partial \mu}$ would be known.

In the next section, restrictions will be found for the scalar functions appearing in Equation (15), by analyzing Equations (13) and (26).

\section{The Expression for $\frac{\partial \Delta H}{\partial \mu}$}

If we substitute Equation (15) in the derivative of $(13)_{1,2}$ with respect to $\mu$, we obtain:

$$
\vartheta_{p, q+1, r, s+1}=\vartheta_{p+2, q, r, s} \quad, \quad \vartheta_{p, q, r+1, s+1}=\frac{\partial}{\partial \lambda} \vartheta_{p+1, q, r, s} .
$$

From Equation $(27)_{1}$, we now obtain:

$$
\vartheta_{p, q, r, s}= \begin{cases}\vartheta_{0, q+\frac{p}{2}, r, s+\frac{p}{2}} & \text { if } p \text { is even } \\ \vartheta_{1, q+\frac{p-1}{2}, r, s+\frac{p-1}{2}} & \text { if } p \text { is odd }\end{cases}
$$

After that, we see that Equation $(27)_{1}$ is satisfied as a consequence of Equation (28).

Let us now focus our attention on Equation $(27)_{2}$; for $p=0,1$, it becomes:

$$
\vartheta_{0, q, r+1, s+1}=\frac{\partial}{\partial \lambda} \vartheta_{1, q, r, s} \quad, \quad \vartheta_{1, q, r+1, s+1}=\frac{\partial}{\partial \lambda} \vartheta_{0, q+1, r, s+1},
$$

where, for $(29)_{2}$, we have used Equation (28) with $p=2$. After that, Equation $(27)_{2}$ will be a consequence of Equations (28) and (29). 
However, we have now to impose the derivative of $(13)_{3}$ with respect to $\mu$, that is:

$$
\frac{\partial^{3} \Delta H}{\partial \mu^{2} \partial \mu_{k}} \mu_{i}+2 \frac{\partial^{3} \Delta H}{\partial \mu^{2} \partial \mu_{k j}} \mu_{j i}+2 \frac{\partial^{3} \Delta H}{\partial \mu^{2} \partial \mu_{k i}} \lambda+2 \frac{\partial^{3} \Delta H}{\partial \mu \partial \mu_{k} \partial \mu_{i j}} \lambda_{j}+\frac{\partial^{3} \Delta H}{\partial \mu^{2} \partial \lambda_{k}} \lambda_{i}+\frac{\partial^{2} \Delta H}{\partial \mu^{2}} \delta^{k i}=0 .
$$

To impose this relation, let us take its derivatives with respect to $\mu_{i_{1}}, \cdots, \mu_{i_{P}}, \mu_{h_{1} k_{1}}, \cdots, \mu_{h_{Q} k_{Q}}, \lambda_{j_{1}}$, $\cdots, \lambda_{j_{R}}$, and let us calculate the result at equilibrium; with some calculations, we obtain:

$$
\begin{aligned}
& 0=(2 Q+R+1) \vartheta_{0, Q, R, s+1}+2 \lambda \vartheta_{0, Q+1, R, s+1}+2 R \vartheta_{1, Q+1, R-1, s}, \\
& 0=(2 Q+R+2) \vartheta_{1, Q, R, s+1}+2 \lambda \vartheta_{1, Q+1, R, s+1}+2 R \vartheta_{0, Q+2, R-1, s+1},
\end{aligned}
$$

with the agreement that the last terms are not present in the case $R=0$. Summarizing the results, we have that Equation (28) gives $\vartheta_{P, Q, R, s}$ in terms of $\vartheta_{0, Q, R, s}$ and $\vartheta_{1, Q, R, s}$, while Equations (29) and (30) give restrictions on $\vartheta_{0, Q, R, s}$ and $\vartheta_{1, Q, R, s}$.

- We want now to impose the further restriction Equation (26). By substituting Equation (15) into Equation (26) and with some calculations, we find:

$$
\begin{gathered}
\frac{\partial^{2} H^{* 0}}{\partial \lambda \partial \mu_{i j}}=\sum_{q}^{0 \cdots \infty} \sum_{r \in I_{0}} \frac{1}{q !} \frac{1}{r !} \vartheta_{1, q, r+1,0} \delta^{\left(i j h_{1} k_{1} \cdots h_{q} k_{q} j_{1} \cdots j_{r}\right)} \mu_{h_{1} k_{1}} \cdots \mu_{h_{q} k_{q}} \lambda_{j_{1}} \cdots \lambda_{j_{r}} . \\
0=\left\{\frac{\partial^{Q}}{\partial \mu_{h_{1} k_{1}} \cdots \partial \mu_{h_{Q} k_{Q}}}\left[2 \mu_{j i} \frac{\partial H^{* 0}}{\partial \mu_{k j}}+2 \lambda \frac{\partial H^{* 0}}{\partial \mu_{k i}}+H^{* 0} \delta^{k i}\right]\right\}_{\lambda_{j}=0, \mu_{i a}=0} . \\
0=\sum_{q}^{0 \cdots \infty} \sum_{r \in I_{1}} \frac{1}{q !} \frac{1}{r !}\left[(2 q+r+2) \vartheta_{1, q, r, 0}+2 \lambda \vartheta_{1, q+1, r, 0}\right] \delta^{\left(a k i h_{1} k_{1} \cdots h_{q} k_{q} j_{1} \cdots j_{r}\right)} \mu_{h_{1} k_{1}} \cdots \mu_{h_{q} k_{q}} \lambda_{j_{1}} \cdots \lambda_{j_{r}} \\
+2 \lambda_{j} \frac{\partial^{2} H^{* 0}\left(\mu_{a b}, \lambda, \lambda_{c}\right)}{\partial \mu_{i j} \partial \mu_{k a}} \\
0=2 \mu_{j i} \frac{\partial^{2} H^{* 0}}{\partial \mu_{k j} \partial \lambda}+2 \lambda \frac{\partial^{2} H^{* 0}}{\partial \mu_{k i} \partial \lambda}+2 \frac{\partial H^{* 0}}{\partial \mu_{k i}}+\lambda_{i} \frac{\partial^{2} H^{* 0}}{\partial \lambda_{k} \partial \lambda}+\delta^{k i} \frac{\partial H^{* 0}}{\partial \lambda}+2 \lambda_{j} \frac{\partial^{2} H^{* 0}}{\partial \mu_{i j} \partial \lambda_{k}} .
\end{gathered}
$$

These are restrictions on $H^{* 0}$.

\section{The Expression for $\Delta H$}

By integrating Equation (15) with respect to $\mu$, we obtain:

$$
\begin{array}{r}
\Delta H=\sum_{p, q, s}^{0 \cdots \infty} \sum_{r \in I_{p}} \frac{1}{p !} \frac{1}{q !} \frac{1}{r !} \frac{1}{(s+1) !} \vartheta_{p, q, r, s}(\lambda) \mu^{s+1} \delta^{\left(i_{1} \cdots i_{p} h_{1} k_{1} \cdots h_{q} k_{q} j_{1} \cdots j_{r}\right)} \\
\mu_{i_{1}} \cdots \mu_{i_{p}} \mu_{h_{1} k_{1}} \cdots \mu_{h_{q} k_{q}} \lambda_{j_{1}} \cdots \lambda_{j_{r}}+\mu H^{* 0}\left(\mu_{a b}, \lambda, \lambda_{c}\right)+\tilde{\tilde{H}}\left(\mu_{a}, \mu_{b c}, \lambda, \lambda_{d}\right) .
\end{array}
$$


By substituting $\Delta H$ from here into $(13)_{1}$ and with an integration, we find:

$$
\begin{array}{r}
\tilde{\tilde{H}}=\sum_{p, q}^{0 \cdots \infty} \sum_{r \in I_{p}} \frac{1}{(p+2) !} \frac{1}{q !} \frac{1}{r !} \vartheta_{p, q+1, r, 0} \delta^{\left(i_{1} \cdots i_{p+2} h_{1} k_{1} \cdots h_{q} k_{q} j_{1} \cdots j_{r}\right)} \\
\mu_{i_{1}} \cdots \mu_{i_{p+2}} \mu_{h_{1} k_{1}} \cdots \mu_{h_{q} k_{q}} \lambda_{j_{1}} \cdots \lambda_{j_{r}}+\frac{1}{2} \mu_{i} \mu_{j} \frac{\partial H^{* 0}}{\partial \mu_{i j}}+\tilde{\tilde{H}}^{i}\left(\mu_{a b}, \lambda, \lambda_{c}\right) \mu_{i}+\tilde{\tilde{H}}^{0}\left(\mu_{a b}, \lambda, \lambda_{c}\right),
\end{array}
$$

where $\tilde{\tilde{H}}^{i}$ and $\tilde{\tilde{H}}^{0}$ arise from the integration.

By substituting $\Delta H$ from Equation (35) into Equation $(13)_{2}$, and by taking into account Equation (36), we find:

$$
\frac{\partial H^{* 0}}{\partial \lambda_{i}}=\frac{\partial \tilde{\tilde{H}}^{i}}{\partial \lambda}
$$

Let us substitute now $\Delta H$ from Equation (35) into (13) $)_{3}$ and take into account Equation (36); we obtain:

$$
2 \mu_{j i} \frac{\partial H^{* 0}}{\partial \mu_{k j}}+2 \lambda \frac{\partial H^{* 0}}{\partial \mu_{k i}}+\lambda_{i} \frac{\partial H^{* 0}}{\partial \lambda_{k}}+\delta^{k i} H^{* 0}+2 \lambda_{j} \frac{\partial \tilde{\tilde{H}}^{k}}{\partial \mu_{i j}}=0
$$

Therefore, the situation is now that Equation (13) is equivalent to Equation (36) (which gives $\tilde{\tilde{H}}$ in terms of $\tilde{\tilde{H}}^{i}\left(\mu_{a b}, \lambda, \lambda_{c}\right)$ and of $\left.\tilde{\tilde{H}}^{0}\left(\mu_{a b}, \lambda, \lambda_{c}\right)\right)$ and to the condition Equations (37) and (38) on $\tilde{\tilde{H}}^{k}$, while $\tilde{\tilde{H}}^{0}$ remains arbitrary, as was obvious, because in Equation (13) it appears only through its derivatives with respect to $\mu$ and $\mu_{k}$, which are zero. We note also that Equation (32) is a particular case of Equation (38), when this last one is calculated in $\lambda_{j}=0$.

A sort of integrability condition can be obtained in the following way: let us take the derivative of Equation (38) with respect to $\mu_{a b}$; let us contract the result with $\lambda_{b}$; and let us take from the resulting equation the skew-symmetric part with respect to $i$ and $a$. In this way, we obtain:

$$
0=2 \mu_{j[i} \frac{\partial^{2} H^{* 0}}{\partial \mu_{a] b} \partial \mu_{k j}} \lambda_{b}+2 \lambda \frac{\partial^{2} H^{* 0}}{\partial \mu_{k[i} \partial \mu_{a] b}} \lambda_{b}+\lambda_{[i} \frac{\partial^{2} H^{* 0}}{\partial \mu_{a] b} \partial \lambda_{k}} \lambda_{b}+\delta^{k[i} \frac{\partial H^{* 0}}{\partial \mu_{a] b}} \lambda_{b} .
$$

To conclude this section, we can say that we have to impose the condition Equations (31)-(34) and (39) on $H^{* 0}\left(\mu_{a b}, \lambda, \lambda_{c}\right)$.

After that, Equations (37) and (38) will give $\tilde{\tilde{H}}^{i}$; we will see that a small further integrability condition will be necessary to this end.

We firstly note that the second term in Equation (39) is zero thanks to Equation (33). We can take the derivative with respect to $\lambda$ of what remains and substitute Equation (31). We obtain a relation and note that:

- Its terms of degree zero in $\mu_{i j}$ and of degree one in $\lambda_{i}$ give $\vartheta_{1,0,1,0}=0$.

- After that, what remains of its term of degree one in $\lambda_{i}$ gives $\vartheta_{1, q+1,1,0}=0$. This result, jointly with the previous one, gives $\vartheta_{1, q, 1,0}=0$. 
- After that, its term of degree zero in $\mu_{i j}$ gives $\vartheta_{1,0, R+3,0}=0$.

- What remains, after a reformatting of its indexes, is equivalent to $\vartheta_{1, q+1, R+3,0}=0$. This result, jointly with the previous one, yields $\vartheta_{1, q, R+3,0}=0$ and $\vartheta_{1, q, R+1,0}=0$.

This result can be rewritten also as: $\quad \vartheta_{1, q, r, 0}=0$,

because the sum of the first and third index must be an even number, so that obviously, we must have $r \geq 1$ in $\vartheta_{1, q, r, 0}$.

By using this condition, we can see that a solution of the condition Equations (31)-(34) and (39) is given by:

$$
\begin{array}{r}
H^{* 0}=\sum_{r \in I_{0}} \frac{1}{(r+2) !} \psi_{0,0, r, 0,0} \delta^{\left(j_{1} \cdots j_{r+2}\right)} \lambda_{j_{1}} \cdots \lambda_{j_{r+2}}-\sum_{r=0}^{\infty} 2 \lambda \frac{2 r+3}{r !}\left(\lambda_{a} \lambda^{a}\right)^{r+1} \beta_{r} \\
+\sum_{r=0}^{\infty} \frac{2 r+3}{r !}\left(\lambda_{a} \lambda^{a}\right)^{r} \beta_{r} \mu_{i k} \lambda^{i} \lambda^{k},
\end{array}
$$

where $\psi_{0,0, r, 0,0}$ and $\beta_{r}$ are two arbitrary sets of constants. In fact,

- it is easy to verify Equation (31), because $H^{* 0}$ given by Equation (41) is the sum of a function not depending on $\lambda$ and of a function not depending on $\mu_{i j}$; moreover, the right-hand side of Equation (31) is zero, thanks to Equation (40).

- It is to verify Equation (32), because $H^{* 0}$ given by Equation (41) becomes zero when calculated in $\lambda_{i}=0$.

- It is to verify Equation (33), thanks to Equation (40) and because $H^{* 0}$ given by Equation (41) is linear in $\mu_{i j}=0$.

- Let us verify Equation (34). By a substitution of $H^{* 0}$ from Equation (41) it becomes:

$$
\begin{gathered}
0=2 \sum_{r=0}^{\infty} \frac{2 r+3}{r !} \beta_{r}\left(\lambda_{a} \lambda^{a}\right)^{r} \lambda^{k} \lambda^{i}+\lambda_{i} \frac{\partial}{\partial \lambda_{k}}\left[-\sum_{r=0}^{\infty} 2 \frac{2 r+3}{r !} \beta_{r}\left(\lambda_{a} \lambda^{a}\right)^{r+1}\right] \\
+\delta^{k i}\left[-\sum_{r=0}^{\infty} 2 \frac{2 r+3}{r !} \beta_{r}\left(\lambda_{a} \lambda^{a}\right)^{r+1}\right]+2 \lambda_{j} \frac{\partial}{\partial \lambda_{k}}\left[\sum_{r=0}^{\infty} \frac{2 r+3}{r !} \beta_{r}\left(\lambda_{a} \lambda^{a}\right)^{r} \lambda^{i} \lambda^{j}\right],
\end{gathered}
$$

which is true, because the sum of the 1st and 4 th term is equal to $\frac{\partial}{\partial \lambda_{k}}\left[2 \sum_{r=0}^{\infty} \frac{2 r+3}{r !} \beta_{r}\left(\lambda_{a} \lambda^{a}\right)^{r} \lambda^{i} \lambda^{j} \lambda_{j}\right]$, while the sum of the 2 nd and 3 rd term is equal to $\frac{\partial}{\partial \lambda_{k}}\left[-\lambda_{i} \sum_{r=0}^{\infty} 2 \frac{2 r+3}{r !} \beta_{r}\left(\lambda_{a} \lambda^{a}\right)^{r+1}\right]$.

- Let us verify Equation (39). By a substitution of $H^{* 0}$ from Equation (41), it becomes:

$$
0=\lambda_{b} \lambda^{[i} \frac{\partial}{\partial \lambda_{k}}\left[\sum_{r=0}^{\infty} \frac{2 r+3}{r !} \beta_{r}\left(\lambda_{c} \lambda^{c}\right)^{r} \lambda^{a]} \lambda^{b}\right]+\lambda_{b} \delta^{k[i}\left[\sum_{r=0}^{\infty} \frac{2 r+3}{r !} \beta_{r}\left(\lambda_{c} \lambda^{c}\right)^{r} \lambda^{a]} \lambda^{b}\right]
$$


In the first term, when we do not take the derivative of $\lambda^{a}$ with respect to $\lambda_{k}$, we obtain zero for the identity $\lambda^{[i} \lambda^{a]}=0$; when we take the derivative of $\lambda^{a}$ with respect to $\lambda_{k}$, we obtain $\lambda_{b} \lambda^{[i} \delta^{a] k} \sum_{r=0}^{\infty} \frac{2 r+3}{r !} \beta_{r}\left(\lambda_{c} \lambda^{c}\right)^{r} \lambda^{b}$, which is the opposite of the second term.

This completes our verification.

We can prove that Equation (41) is the unique solution of our conditions, but the passages are very long and boring; so we avoid reporting them for the sake of brevity; the interested reader can ask for this proof, and we will send it to him or her. Only to mention briefly the strategy of the proof, we say that Equation (31) can be used, with some passages, to obtain $H^{* 0}$, except for an arbitrary function $\tilde{H}^{* 02}\left(\mu_{i j}, \lambda_{k}\right)$. After that, Equation (33) will give the expression of $\lambda_{j} \frac{\partial^{2} \tilde{H}^{* 02}}{\partial \mu_{i j} \partial \mu_{k a}}$.

Subsequently, Equation (34) will give the expression of $\frac{\partial \tilde{H}^{* 02}}{\partial \mu_{k i}}+\lambda_{j} \frac{\partial^{2} \tilde{H}^{* 02}}{\partial \mu_{i j} \partial \lambda_{k}}$, and Equations (32), (39) will give other conditions on $\tilde{H}^{* 02}$. By solving all of these conditions and substituting the resulting expression of $\tilde{H}^{* 02}$ into the above-mentioned $H^{* 0}$, we obtain Equation (41).

\section{Solution of the Conditions on $\tilde{\tilde{H}}^{i}$}

Let us firstly change the unknown function from $\tilde{\tilde{H}}^{k}$ to $\tilde{\tilde{H}}^{* k}$ defined by:

$$
\begin{aligned}
& \tilde{\tilde{H}}^{k}=\tilde{\tilde{H}}^{* k}+\sum_{r \in I_{0}} \frac{1}{(r+1) !}\left[\lambda \delta^{\left(k j_{1} \cdots j_{r+1}\right)}-\frac{1}{2} \frac{r+3}{r+2} \delta^{\left(k i j j_{1} \cdots j_{r+1}\right)} \mu_{i j}\right] \psi_{0,0, r, 0,0} \lambda_{j_{1}} \cdots \lambda_{j_{r+1}} \\
& +\frac{\partial}{\partial \lambda_{k}}\left[\sum_{r=0}^{\infty} \frac{2 r+3}{r !} \beta_{r}\left(\lambda_{a} \lambda^{a}\right)^{r}\left(\lambda \mu_{b c} \lambda^{b} \lambda^{c}-\lambda^{2} \lambda_{b} \lambda^{b}\right)\right] \\
& -\mu^{k d} \lambda_{d}\left(\mu_{b c} \lambda^{b} \lambda^{c}\right) \sum_{r=2}^{\infty} \frac{2 r+3}{r !} \beta_{r}\left(\lambda_{a} \lambda^{a}\right)^{r-1}-\frac{1}{4} \lambda^{k}\left(\mu_{b c} \lambda^{b} \lambda^{c}\right)^{2} \sum_{r=2}^{\infty}(2 r-3) \frac{2 r+3}{r !} \beta_{r}\left(\lambda_{a} \lambda^{a}\right)^{r-2} \\
& -\lambda^{k}\left(\mu_{b d} \mu_{d c} \lambda^{b} \lambda^{c}\right) \sum_{r=2}^{\infty} \frac{2 r+3}{r !} \beta_{r}\left(\lambda_{a} \lambda^{a}\right)^{r-1} .
\end{aligned}
$$

By substituting $\tilde{\tilde{H}}^{k}$ from Equation (42) and $H^{* 0}$ from Equation (41) into Equations (37) and (38), these equations are transformed respectively into:

$$
\begin{gathered}
\frac{\partial}{\partial \lambda} \tilde{\tilde{H}}^{* i}=0 \\
0=2 \lambda_{j} \frac{\partial \tilde{\tilde{H}}^{* k}}{\partial \mu_{i j}}+\left[\delta^{k i}\left(\mu_{b c} \lambda^{b} \lambda^{c}\right)+4 \lambda^{(k} \mu^{i) j} \lambda_{j}\right]\left(3 \beta_{0}+5 \beta_{1} \lambda_{a} \lambda^{a}\right)+10 \lambda^{i} \lambda^{k}\left(\mu_{b c} \lambda^{b} \lambda^{c}\right) \beta_{1} .
\end{gathered}
$$

A further refinement of the situation can be obtained with another change of the unknown function from $\tilde{\tilde{H}}^{* k}$ to $\tilde{\tilde{H}}^{* * k}$ defined by:

$$
\tilde{\tilde{H}}^{* k}=\tilde{\tilde{H}}^{* * k}-\frac{5}{4} \beta_{1}\left\{4\left(\mu_{b c} \lambda^{b} \lambda^{c}\right) \mu^{k d} \lambda_{d}+\lambda^{k}\left[\left(\mu_{b c} \mu^{b c}\right)\left(\lambda_{a} \lambda^{a}\right)+2 \lambda^{a} \lambda^{b} \mu_{a c} \mu_{c b}\right]\right\} .
$$

By using this, Equation (44) becomes:

$$
0=2 \lambda_{j} \frac{\partial \tilde{\tilde{H}}^{* * k}}{\partial \mu_{i j}}+3 \beta_{0}\left[\delta^{k i}\left(\mu_{b c} \lambda^{b} \lambda^{c}\right)+4 \lambda^{(k} \mu^{i) j} \lambda_{j}\right]
$$


We can now prove that, as a consequence of this equation, we have:

$$
\beta_{0}=0
$$

Solution of the Conditions on $\tilde{\tilde{H}}^{* * k}$

Let us consider the Taylor expansion of $\tilde{\tilde{H}}^{* * k}$ around the state with $\mu^{i j}=0$; Equation (46) at the order one with respect to this state is:

$$
0=2 \lambda_{j} \frac{\partial \tilde{\tilde{H}}_{2}^{* * k}}{\partial \mu_{i j}}+3 \beta_{0}\left[\delta^{k i}\left(\mu_{b c} \lambda^{b} \lambda^{c}\right)+4 \lambda^{(k} \mu^{i) j} \lambda_{j}\right]
$$

where $\tilde{\tilde{H}}_{2}^{* * k}$ is the homogeneous part of $\tilde{\tilde{H}}^{* * k}$ of second degree with respect to $\mu^{i j}$. Thanks to the representation theorems, it has the form:

$$
\begin{aligned}
& \tilde{\tilde{H}}_{2}^{* * k}=f_{1}\left(G_{0}\right) \mu^{k a} \mu^{a b} \lambda_{b}+f_{2}\left(G_{0}\right) \mu^{l l} \mu^{k a} \lambda_{a}+f_{3}\left(G_{0}\right)\left(\mu_{b c} \lambda^{b} \lambda^{c}\right) \mu^{k a} \lambda_{a} \\
& \left.+\lambda^{k}\left[f_{4}\left(G_{0}\right)\left(\mu^{l l}\right)^{2}+f_{5}\left(G_{0}\right)\left(\mu_{b c} \lambda^{b} \lambda^{c}\right)^{2}+f_{6}\left(G_{0}\right)\left(\mu_{b c} \lambda^{b} \lambda^{c}\right) \mu^{l l}+f_{7}\left(G_{0}\right) \mu_{b c} \mu^{b c}+f_{8}\left(G_{0}\right) \mu_{b a} \mu_{a c} \lambda^{b} \lambda^{c}\right)\right],
\end{aligned}
$$

where $G_{0}=\lambda_{a} \lambda^{a}$. By substituting this into Equation (48), we obtain:

$$
\begin{array}{r}
0=2 \lambda_{j}\left\{f_{1} \delta^{k(i} \mu^{j) b} \lambda_{b}+f_{1} \mu^{k(i} \lambda^{j)}+f_{2} \delta^{i j} \mu^{k b} \lambda_{b}+f_{2} \mu^{l l} \delta^{k(i} \lambda^{j)}+f_{3} \lambda^{i} \lambda^{j} \mu^{k b} \lambda_{b}+f_{3}\left(\mu_{b c} \lambda^{b} \lambda^{c}\right) \delta^{k(i} \lambda^{j)}\right. \\
\left.+\lambda^{k}\left[2 f_{4} \mu^{l l} \delta^{i j}+2 f_{5}\left(\mu_{b c} \lambda^{b} \lambda^{c}\right) \lambda^{i} \lambda^{j}+f_{6} \lambda^{i} \lambda^{j} \mu^{l l}+f_{6}\left(\mu_{b c} \lambda^{b} \lambda^{c}\right) \delta^{i j}+2 f_{7} \mu^{i j}+2 f_{8} \lambda^{(i} \mu^{j) b} \lambda_{b}\right]\right\} \\
+3 \beta_{0}\left[\delta^{k i}\left(\mu_{b c} \lambda^{b} \lambda^{c}\right)+4 \lambda^{(k} \mu^{i) j} \lambda_{j}\right]
\end{array}
$$

that is,

$$
\begin{array}{r}
0=f_{1} \delta^{k i}\left(\mu_{b c} \lambda^{b} \lambda^{c}\right)+2 f_{1} \lambda^{(k} \mu^{i) b} \lambda_{b}+f_{1} \mu^{k i} G_{0}+2 f_{2} \lambda^{i} \mu^{k b} \lambda_{b}+f_{2} \mu^{l l} \delta^{k i} G_{0}+f_{2} \mu^{l l} \lambda^{k} \lambda^{i} \\
+2 f_{3} G_{0} \lambda^{i} \mu^{k b} \lambda_{b}+f_{3}\left(\mu_{b c} \lambda^{b} \lambda^{c}\right) \delta^{k i} G_{0}+f_{3}\left(\mu_{b c} \lambda^{b} \lambda^{c}\right) \lambda^{k} \lambda^{i} \\
+\lambda^{k}\left[4 f_{4} \mu^{l l} \lambda^{i}+4 f_{5}\left(\mu_{b c} \lambda^{b} \lambda^{c}\right) G_{0} \lambda^{i}+2 f_{6} G_{0} \lambda^{i} \mu^{l l}+2 f_{6}\left(\mu_{b c} \lambda^{b} \lambda^{c}\right) \lambda^{i}+4 f_{7} \mu^{i j} \lambda_{j}+2 f_{8} \lambda^{i}\left(\mu_{b c} \lambda^{b} \lambda^{c}\right)\right. \\
\left.+2 f_{8} G_{0} \mu^{i b} \lambda_{b}\right]+3 \beta_{0}\left[\delta^{k i}\left(\mu_{b c} \lambda^{b} \lambda^{c}\right)+4 \lambda^{(k} \mu^{i) j} \lambda_{j}\right] .
\end{array}
$$

The skew-symmetric part of this relation, with respect to $i$ and $k$, is:

$$
0=\lambda^{[k} \mu^{i] b} \lambda_{b}\left(-2 f_{2}-2 f_{3} G_{0}+4 f_{7}+2 f_{8} G_{0}\right),
$$

from which we obtain:

$$
4 f_{7}=2 f_{2}+2 f_{3} G_{0}-2 f_{8} G_{0}
$$

By taking into account this value of $f_{7}$, the remaining part of our condition becomes:

$$
\begin{aligned}
0=\quad & \delta^{k i}\left[f_{2} \mu^{l l} G_{0}+\left(f_{1}+f_{3} G_{0}+3 \beta_{0}\right)\left(\mu_{b c} \lambda^{b} \lambda^{c}\right)\right]+f_{1} \mu^{k i} G_{0} \\
& +\lambda^{(k} \mu^{i) b} \lambda_{b}\left(2 f_{1}+4 f_{2}+4 f_{3} G_{0}+12 \beta_{0}\right) \\
& +\lambda^{k} \lambda^{i}\left[\mu^{l l}\left(f_{2}+4 f_{4}+2 f_{6} G_{0}\right)+\left(\mu_{b c} \lambda^{b} \lambda^{c}\right)\left(f_{3}+4 f_{5} G_{0}+2 f_{6}+2 f_{8}\right)\right] .
\end{aligned}
$$


In this relation, the coefficients of $\mu^{k i}$ and $\delta^{k i} \mu^{l l}$ give, respectively, $f_{1}=0$ and $f_{2}=0$. After that, the coefficient of $\delta^{k i}\left(\mu_{b c} \lambda^{b} \lambda^{c}\right)$ gives $f_{3} G_{0}+3 \beta_{0}=0$, which, calculated in $\lambda_{j}=0$, gives the above-mentioned Equation (47).

This result transforms Equation (46) into:

$$
0=2 \lambda_{j} \frac{\partial \tilde{\tilde{H}}^{* * k}}{\partial \mu_{i j}}
$$

Now, we proceed to find the general solution of this last equation, and we prove that it is:

$$
\tilde{\tilde{H}}^{* * k}=\lambda^{k} F\left(G_{0}, G_{1}, G_{2}\right) \text {, }
$$

where:

$$
G_{1}=G_{0} \delta^{b c} \mu_{b c}-\mu_{b c} \lambda^{b} \lambda^{c}, \quad G_{2}=G_{0} \mu^{b c} \mu_{b c}-2 \mu_{b c} \mu_{c a} \lambda^{b} \lambda^{a}+2\left(\delta^{b c} \mu_{b c}\right)\left(\mu_{b c} \lambda^{b} \lambda^{c}\right)-G_{0}\left(\delta^{b c} \mu_{b c}\right)^{2}
$$

and $F$ is an arbitrary function of its variables.

In fact, if $\lambda_{j}=0$, from the representation theorems, we know that $\tilde{\tilde{H}}^{* * k}=0$, just as in Equations (51) and (50), is an identity.

If $\lambda_{j} \neq 0$, we can define the projector into the subspace orthogonal to $\lambda_{j}$, that is:

$$
h^{i j}=\delta^{i j}-\frac{1}{G_{0}} \lambda^{i} \lambda^{j}
$$

from which it follows $h^{i j} \lambda_{j}=0$, as is obvious. By taking as independent variables $\lambda^{i}, \tilde{\mu}=\mu_{b c} \lambda^{b} \lambda^{c}$, $\tilde{\mu}^{i}=h^{i j} \mu_{j a} \lambda^{a}, \tilde{\mu}^{i j}=h^{i a} \mu_{a b} h^{b j}$,

Equation (50) becomes:

$$
0=2 \lambda_{j}\left(\frac{\partial \tilde{\tilde{H}}^{* * k}}{\partial \tilde{\mu}} \lambda^{i} \lambda^{j}+\frac{\partial \tilde{\tilde{H}}^{* * k}}{\partial \tilde{\mu}^{b}} h^{b(j} \lambda^{i)}+\frac{\partial \tilde{\tilde{H}}^{* * k}}{\partial \tilde{\mu}^{a b}} h^{a(i} h^{j) b}\right)=2 G_{0} \frac{\partial \tilde{\tilde{H}}^{* * k}}{\partial \tilde{\mu}} \lambda^{i}+\frac{\partial \tilde{\tilde{H}}^{* * k}}{\partial \tilde{\mu}^{b}} G_{0} h^{b i} .
$$

By contracting this relation with $\lambda_{i}$ and with $h_{i a}$, we obtain, respectively:

$$
\frac{\partial \tilde{\tilde{H}}^{* * k}}{\partial \tilde{\mu}}=0 \quad, \quad \frac{\partial \tilde{\tilde{H}}^{* * k}}{\partial \tilde{\mu}^{a}}=0
$$

It follows that $\tilde{\tilde{H}}^{* * k}$ may depend only on $\lambda^{i}$ and $\tilde{\mu}^{i j}$. However, $\tilde{\mu}^{i j} \lambda_{j}=0$, so that, for the representation theorems, we have that $\tilde{\tilde{H}}^{* * k}$ is proportional to $\lambda^{k}$, as in Equation (51); moreover, the coefficient $F$ can be a scalar function of $G_{0}, Q_{1}=\delta_{i j} \tilde{\mu}^{i j}, Q_{2}=\delta_{i j} \tilde{\mu}^{i a} \tilde{\mu}^{a j}$.

Now, we have:

$$
\begin{gathered}
Q_{1}=\delta_{i j} \tilde{\mu}^{i j}=h_{i j} \mu^{i j}=\delta_{i j} \mu^{i j}-\frac{1}{G_{0}} \lambda_{i} \lambda_{j} \mu^{i j}, \\
Q_{2}=\delta_{i j} h^{i b} \mu_{b c} h^{c a} h^{a d} \mu_{d e} h^{e j}=h_{b e} \mu^{b c} h_{c d} \mu^{d e}=\delta_{b e} \mu^{e c} \delta_{c d} \mu^{d e}-\frac{2}{G_{0}} \delta_{b e} \mu^{b c} \lambda_{c} \lambda_{d} \mu^{d e}+\left(\frac{1}{G_{0}}\right)^{2}\left(\mu_{b c} \lambda^{b} \lambda^{c}\right)^{2} .
\end{gathered}
$$

However, an arbitrary function of $G_{0}, Q_{1}, Q_{2}$ is also an arbitrary function of $G_{0}, Q_{1}$ and of:

$$
Q_{2}-\left(Q_{1}\right)^{2}=\mu^{e c} \mu_{e c}-\frac{2}{G_{0}} \mu^{d e} \mu^{e c} \lambda_{d} \lambda_{c}+\frac{2}{G_{0}}\left(\delta_{i j} \mu^{i j}\right)\left(\mu_{a b} \lambda^{a} \lambda^{b}\right)-\left(\delta_{i j} \mu^{i j}\right)^{2}
$$

and an arbitrary function of $G_{0}, Q_{1}, Q_{2}-\left(Q_{1}\right)^{2}$ is also an arbitrary function of $G_{0}, Q_{1} G_{0},\left[Q_{2}-\left(Q_{1}\right)^{2}\right] G_{0}$, and this completes the proof of Equation $(51)_{2,3}$. These last passages have been done with the end result being to have a function defined also in $\lambda^{j}=0$, without going too far from equilibrium. 


\section{Conclusions}

We can now collect all of our results. By substituting $\tilde{\tilde{H}}_{2}^{* * k}$ from Equation (51) into Equation (45), we obtain $\tilde{\tilde{H}}^{* k}$; by substituting this and Equation (47) into Equation (42), we obtain the expression of $\tilde{\tilde{H}}^{k}$. Thanks to this expression and Equation (41), taking also into account Equation (47), we can rewrite the expression for $\tilde{\tilde{H}}$ in Equation (36); finally, we can substitute this new expression and that of Equation (41) for $H^{* 0}$ into Equation (35). In this way, we obtain:

$$
\begin{aligned}
& \Delta H=\sum_{p, q, s}^{0 \cdots \infty} \sum_{r \in I_{p}} \frac{1}{p !} \frac{1}{q !} \frac{1}{r !} \frac{1}{(s+1) !} \vartheta_{p, q, r, s}(\lambda) \mu^{s+1} \delta^{\left(i_{1} \cdots i_{p} h_{1} k_{1} \cdots h_{q} k_{q} j_{1} \cdots j_{r}\right)} \\
& \mu_{i_{1}} \cdots \mu_{i_{p}} \mu_{h_{1} k_{1}} \cdots \mu_{h_{q} k_{q}} \lambda_{j_{1}} \cdots \lambda_{j_{r}}+ \\
& +\mu\left\{\sum_{r \in I_{0}} \frac{1}{(r+2) !} \psi_{0,0, r, 0,0} \delta^{\left(j_{1} \cdots j_{r+2}\right)} \lambda_{j_{1}} \cdots \lambda_{j_{r+2}}-\sum_{r=1}^{\infty} 2 \lambda \frac{2 r+3}{r !}\left(\lambda_{a} \lambda^{a}\right)^{r+1} \beta_{r}+\right. \\
& \left.+\sum_{r=1}^{\infty} \frac{2 r+3}{r !}\left(\lambda_{a} \lambda^{a}\right)^{r} \beta_{r} \mu_{i k} \lambda^{i} \lambda^{k}\right\}+ \\
& +\sum_{p, q}^{0 \cdots \infty} \sum_{r \in I_{p}} \frac{1}{(p+2) !} \frac{1}{q !} \frac{1}{r !} \vartheta_{p, q+1, r, 0} \delta^{\left(i_{1} \cdots i_{p+2} h_{1} k_{1} \cdots h_{q} k_{q} j_{1} \cdots j_{r}\right)} \\
& \mu_{i_{1}} \cdots \mu_{i_{p+2}} \mu_{h_{1} k_{1}} \cdots \mu_{h_{q} k_{q}} \lambda_{j_{1}} \cdots \lambda_{j_{r}}+\frac{1}{2} \mu_{i} \mu_{j} \sum_{r=1}^{\infty} \frac{2 r+3}{r !}\left(\lambda_{a} \lambda^{a}\right)^{r} \beta_{r} \lambda^{i} \lambda^{j}+ \\
& +\mu_{i}\left\{\lambda^{i} F\left(G_{0}, G_{1}, G_{2}\right)-\frac{5}{4} \beta_{1}\left[4\left(\mu_{b c} \lambda^{b} \lambda^{c}\right) \mu^{i d} \lambda_{d}+\lambda^{i}\left(\left(\mu_{b c} \mu^{b c}\right)\left(\lambda_{a} \lambda^{a}\right)+2 \lambda^{a} \lambda^{b} \mu_{a c} \mu_{c b}\right)\right]+\right. \\
& +\sum_{r \in I_{0}} \frac{1}{(r+1) !}\left[\lambda \delta^{\left(i j_{1} \cdots j_{r+1}\right)}-\frac{1}{2} \frac{r+3}{r+2} \delta^{\left(i k j j_{1} \cdots j_{r+1}\right)} \mu_{k j}\right] \psi_{0,0, r, 0,0} \lambda_{j_{1}} \cdots \lambda_{j_{r+1}}+ \\
& +\frac{\partial}{\partial \lambda_{i}}\left[\sum_{r=1}^{\infty} \frac{2 r+3}{r !} \beta_{r}\left(\lambda_{a} \lambda^{a}\right)^{r}\left(\lambda \mu_{b c} \lambda^{b} \lambda^{c}-\lambda^{2} \lambda_{b} \lambda^{b}\right)\right]+ \\
& -\mu^{i d} \lambda_{d}\left(\mu_{b c} \lambda^{b} \lambda^{c}\right) \sum_{r=2}^{\infty} \frac{2 r+3}{r !} \beta_{r}\left(\lambda_{a} \lambda^{a}\right)^{r-1}-\frac{1}{4} \lambda^{i}\left(\mu_{b c} \lambda^{b} \lambda^{c}\right)^{2} \sum_{r=2}^{\infty}(2 r-3) \frac{2 r+3}{r !} \beta_{r}\left(\lambda_{a} \lambda^{a}\right)^{r-2}+ \\
& \left.-\lambda^{i}\left(\mu_{b d} \mu_{d c} \lambda^{b} \lambda^{c}\right) \sum_{r=2}^{\infty} \frac{2 r+3}{r !} \beta_{r}\left(\lambda_{a} \lambda^{a}\right)^{r-1}\right\}+\tilde{\tilde{H}}^{0}\left(\mu_{a b}, \lambda, \lambda_{c}\right) .
\end{aligned}
$$

We recall that in this expression, $F\left(G_{0}, G_{1}, G_{2}\right)$ is an arbitrary function, $\psi_{0,0, r, 0,0}$ and $\beta_{r}$ are arbitrary constants, while $\vartheta_{p, q, r, s}(\lambda)$ are constrained by Equations (28)-(30), (40), (16) 1 and (16) 2 . The presence of the arbitrary function $\tilde{H}^{0}\left(\mu_{a b}, \lambda, \lambda_{c}\right)$ is obvious, since it is not constrained by Equation (13), because it does not depend on $\mu$, nor on $\mu_{k}$. Consequently, it is not necessary to impose the condition $\tilde{\tilde{H}}^{0}\left(0_{a b}, \lambda, 0_{c}\right)=0$, which comes out from Equations (14), (16) $)_{2}$ and (54).

The sum of the expression Equation (54) for $\Delta H$ and of the expression Equation (10) for $H_{1}$ gives the general solution for the unknown function $H$. Let us substitute it into the equations:

$$
F^{k i j}=\frac{\partial^{2} H}{\partial \mu_{k} \partial \mu_{i j}} \quad, \quad G^{k i}=\frac{\partial^{2} H}{\partial \mu_{k} \partial \lambda_{i}}
$$


which are a subset of the Equation (3). We obtain that $F^{k i j}-\Delta F^{k i j}$ and $G^{k i}-\Delta G^{k i}$ are symmetric tensors, with $\Delta F^{k i j}$ and $\Delta G^{k i}$ defined by:

$$
\begin{aligned}
& \Delta F^{k i j}= \lambda^{k} \frac{\partial F\left(G_{0}, G_{1}, G_{2}\right)}{\partial \mu_{i j}}-\frac{5}{4} \beta_{1}\left[4\left(\mu_{b c} \lambda^{b} \lambda^{c}\right) \delta^{k(i} \lambda^{j)}+\lambda^{k}\left(2 \mu^{i j}\left(\lambda_{a} \lambda^{a}\right)-4 \lambda^{(i} \mu^{j) b} \lambda_{b}\right)\right] \\
&+2 \sum_{r=1}^{\infty} \frac{2 r+3}{r !} \beta_{r}\left(\lambda_{a} \lambda^{a}\right)^{r} \lambda \delta^{k(i} \lambda^{j)}-\delta^{k(i} \lambda^{j)}\left(\mu_{b c} \lambda^{b} \lambda^{c}\right) \sum_{r=2}^{\infty} \frac{2 r+3}{r !} \beta_{r}\left(\lambda_{a} \lambda^{a}\right)^{r-1} \\
& \Delta G^{k i}= \sum_{r=1}^{\infty} \frac{2 r+3}{r !}\left(\lambda_{a} \lambda^{a}\right)^{r} \beta_{r} \lambda^{k} \mu^{i}+\lambda^{k} \frac{\partial F}{\partial G_{1}} \frac{\partial G_{1}}{\partial \lambda_{i}}+\lambda^{k} \frac{\partial F}{\partial G_{2}} \frac{\partial G_{2}}{\partial \lambda_{i}}-5 \beta_{1} \lambda^{k} \mu^{i c} \mu^{c b} \lambda_{b} \\
&-2 \lambda^{i} \mu^{k d} \lambda_{d}\left(\mu_{b c} \lambda^{b} \lambda^{c}\right) \sum_{r=2}^{\infty} \frac{2 r+3}{r !}(r-1) \beta_{r}\left(\lambda_{a} \lambda^{a}\right)^{r-2} \\
&-\lambda^{k} \mu^{i d} \lambda_{d}\left(\mu_{b c} \lambda^{b} \lambda^{c}\right) \sum_{r=2}^{\infty}(2 r-3) \frac{2 r+3}{r !} \beta_{r}\left(\lambda_{a} \lambda^{a}\right)^{r-2}-2 \lambda^{k} \mu^{i d} \mu^{d c} \lambda_{c} \sum_{r=2}^{\infty} \frac{2 r+3}{r !} \beta_{r}\left(\lambda_{a} \lambda^{a}\right)^{r-1} .
\end{aligned}
$$

It follows that the eventual non-symmetric parts for $F^{k i j}$ and $G^{k i}$ may come only from $\Delta F^{k i j}$ and $\Delta G^{k i}$, respectively. However, from $(51)_{2,3}$, we see that $\frac{\partial G_{1}}{\partial \mu_{i j}}$ is a tensor at least of second order with respect to equilibrium and $\frac{\partial G_{2}}{\partial \mu_{i j}}$ is a tensor at least of third order with respect to equilibrium. Consequently, from Equation (56), it is clear that $F^{k i j}$ up to second order with respect to equilibrium is a symmetric tensor; its eventual non-symmetric parts may appear only from the third order with respect to equilibrium. This result is different from its counterpart in [24], where a non-symmetric part appeared also at first order with respect to equilibrium. We shall see in Appendix 3 that from the equations of that paper, it follows that this non-symmetric part is proportional to a constant; consequently, here, we have proven that this constant is zero and that this further constraint follows by imposing the equations up to order higher than one with respect to equilibrium. This is not a problem, because the authors of [24] assumed (for example, in the first three lines of Subsection 7.2) that the integration constants vanish and furnished reasons for this assumption based on the kinetic theory approach.

Similarly, from $(51)_{2,3}$, we see that $\frac{\partial G_{1}}{\partial \lambda_{i}}$ is a tensor of second order with respect to equilibrium and $\frac{\partial G_{2}}{\partial \lambda_{i}}$ is a tensor at least of third order with respect to equilibrium. Consequently, from Equation (57), it is clear that $G^{k i}$ up to second order with respect to equilibrium is a symmetric tensor; its eventual non-symmetric parts may appear only from the third order with respect to equilibrium. This result agrees with its counterpart in [24].

\section{Acknowledgments}

We thank M. Sugiyama, T. Ruggeri and also four anonymous referees for suggestions, which were useful to improve the results of the present article.

\section{Author Contributions}

Each author contributed equally to the development work. 


\section{Conflicts of Interest}

The authors declare no conflict of interest.

\section{Appendix 1. The Particular Solution $H=H_{1}$}

Let us prove that $H=H_{1}$, with $H_{1}$ given by Equation (10) and $\psi_{n}$ constrained by Equation(11), is a particular solution of Equations (8) and (9).

In fact, by substituting Equation (10) in $(8)_{1}$, we obtain:

$$
\frac{\partial^{r+p+1}}{\partial \lambda^{r} \partial \mu^{p+1}}\left[\left(\frac{-1}{2 \lambda}\right)^{q+1+\frac{p+r}{2}} \psi_{\frac{p+r}{2}}\right]=\frac{\partial^{r+p+2}}{\partial \lambda^{r} \partial \mu^{p+2}}\left[\left(\frac{-1}{2 \lambda}\right)^{q+\frac{p+2+r}{2}} \psi_{\frac{p+2+r}{2}}\right]
$$

which surely holds because $\psi_{\frac{p+r}{2}}=\frac{\partial}{\partial \mu} \psi_{\frac{p+r}{2}+1}$, thanks to Equation (11).

By substituting Equation (10) into $(8)_{2}$, we obtain:

$$
\frac{\partial^{r+p+2}}{\partial \lambda^{r+1} \partial \mu^{p+1}}\left[\left(\frac{-1}{2 \lambda}\right)^{q+\frac{p+r+1}{2}} \psi_{\frac{p+r+1}{2}}\right]=\frac{\partial^{r+p+2}}{\partial \lambda^{r+1} \partial \mu^{p+1}}\left[\left(\frac{-1}{2 \lambda}\right)^{q+\frac{p+1+r}{2}} \psi_{\frac{p+1+r+}{2}}\right]
$$

which is an evident identity.

It is more delicate to verify Equation (9). To do it, let us substitute Equation (9) with its derivatives with respect to $\mu_{i_{1}}, \cdots, \mu_{i_{P}}, \mu_{h_{1} k_{1}}, \cdots, \mu_{h_{Q} k_{Q}}, \lambda_{j_{1}}, \cdots, \lambda_{j_{R}}$; let us substitute Equation (10) into the resulting equation, and let us calculate the last form at equilibrium. We obtain:

$$
\begin{aligned}
& 0=P \delta^{i \overline{i_{1}}} \delta^{\left(\overline{i_{2} \cdots i_{P}} k h_{1} k_{1} \cdots h_{Q} k_{Q} j_{1} \cdots j_{R}\right)} \frac{(P+2 Q+R+1) ! !}{P+2 Q+R+1} \frac{\partial^{R+P+1}}{\partial \lambda^{R} \partial \mu^{P+1}}\left[\left(\frac{-1}{2 \lambda}\right)^{Q+\frac{P+R}{2}} \psi_{\frac{P+R}{2}}\right] \\
& +2 Q \delta^{i \overline{h_{1}}} \delta^{\left(\overline{k_{1} h_{2} k_{2} \cdots h_{Q} k_{Q}} k i_{1} \cdots i_{P} j_{1} \cdots j_{R}\right)} \frac{(P+2 Q+R+1) ! !}{P+2 Q+R+1} \frac{\partial^{R+P+1}}{\partial \lambda^{R} \partial \mu^{P+1}}\left[\left(\frac{-1}{2 \lambda}\right)^{Q+\frac{P+R}{2}} \psi_{\frac{P+R}{2}}\right] \\
& +2 \lambda \delta^{\left(k i h_{1} k_{1} \cdots h_{Q} k_{Q} i_{1} \cdots i_{P} j_{1} \cdots j_{R}\right)}(P+2 Q+R+1) ! ! \frac{\partial^{R+P+1}}{\partial \lambda^{R} \partial \mu^{P+1}}\left[\left(\frac{-1}{2 \lambda}\right)^{Q+1+\frac{P+R}{2}} \psi_{\frac{P+R}{2}}\right] \\
& +2 R \delta^{\left(k i h_{1} k_{1} \cdots h_{Q} k_{Q} i_{1} \cdots i_{P} j_{1} \cdots j_{R}\right)}(P+2 Q+R+1) ! ! \frac{\partial^{R+P}}{\partial \lambda^{R-1} \partial \mu^{P+1}}\left[\left(\frac{-1}{2 \lambda}\right)^{Q+1+\frac{P+R}{2}} \psi_{\frac{P+R}{2}}\right] \\
& +R \delta^{i \overline{j_{1}}} \delta^{\left(\overline{j_{2} \cdots j_{R}} k h_{1} k_{1} \cdots h_{Q} k_{Q} i_{1} \cdots i_{P}\right)} \frac{(P+2 Q+R+1) ! !}{P+2 Q+R+1} \frac{\partial^{R+P+1}}{\partial \lambda^{R} \partial \mu^{P+1}}\left[\left(\frac{-1}{2 \lambda}\right)^{Q+\frac{P+R}{2}} \psi_{\frac{P+R}{2}}\right] \\
& \quad+\delta^{k i} \delta^{\left(i_{1} \cdots i_{P} h_{1} k_{1} \cdots h_{Q} k_{Q} j_{1} \cdots j_{R}\right)} \frac{(P+2 Q+R+1) ! !}{P+2 Q+R+1} \frac{\partial^{R+P+1}}{\partial \lambda^{R} \partial \mu^{P+1}}\left[\left(\frac{-1}{2 \lambda}\right)^{Q+\frac{P+R}{2}} \psi_{\frac{P+R}{2}}\right],
\end{aligned}
$$

where overlined indexes denote symmetrization over those indexes, after that, the other one (round brackets around indexes) has been taken. 
Now, the first, second, fifth and sixth term can be put together, so that the above expression becomes:

$$
\begin{array}{r}
0=\delta^{i \overline{i_{1}}} \delta^{\left(\overline{i_{2} \cdots i_{P} k h_{1} k_{1} \cdots h_{Q} k_{Q} j_{1} \cdots j_{R}}\right)}(P+2 Q+R+1) ! ! \frac{\partial^{R+P+1}}{\partial \lambda^{R} \partial \mu^{P+1}}\left[\left(\frac{-1}{2 \lambda}\right)^{Q+\frac{P+R}{2}} \psi_{\frac{P+R}{2}}\right] \\
+(P+2 Q+R+1) ! ! \delta^{\left(k i h_{1} k_{1} \cdots h_{Q} k_{Q} i_{1} \cdots i_{P} j_{1} \cdots j_{R}\right)}\left\{2 \lambda \frac{\partial^{R+P+1}}{\partial \lambda^{R} \partial \mu^{P+1}}\left[\left(\frac{-1}{2 \lambda}\right)^{Q+1+\frac{P+R}{2}} \psi_{\frac{P+R}{2}}\right]\right. \\
\left.+2 R \frac{\partial^{R+P}}{\partial \lambda^{R-1} \partial \mu^{P+1}}\left[\left(\frac{-1}{2 \lambda}\right)^{Q+1+\frac{P+R}{2}} \psi_{\frac{P+R}{2}}\right]\right\},
\end{array}
$$

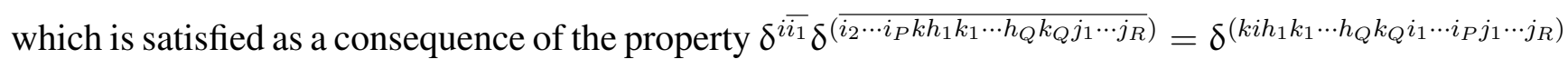
and of the identity:

$$
\begin{array}{r}
\frac{\partial^{R}}{\partial \lambda^{R}}\left[\left(\frac{-1}{2 \lambda}\right)^{Q+\frac{P+R}{2}} \psi_{\frac{P+R}{2}}\right]=\frac{\partial^{R}}{\partial \lambda^{R}}\left[-2 \lambda\left(\frac{-1}{2 \lambda}\right)^{Q+1+\frac{P+R}{2}} \psi_{\frac{P+R}{2}}\right] \\
=-2 \lambda \frac{\partial^{R}}{\partial \lambda^{R}}\left[\left(\frac{-1}{2 \lambda}\right)^{Q+1+\frac{P+R}{2}} \psi_{\frac{P+R}{2}}\right]-2 R \frac{\partial^{R-1}}{\partial \lambda^{R-1}}\left[\left(\frac{-1}{2 \lambda}\right)^{Q+1+\frac{P+R}{2}} \psi_{\frac{P+R}{2}}\right] .
\end{array}
$$

This completes the proof that $H=H_{1}$ is a particular solution of Equations (8) and (9).

\section{Appendix 2. Proof of Property 1}

Let us prove it with the iterative procedure, and let $\Delta H^{n}$ denote the homogeneous part of $\Delta H$ of order $n$ with respect to equilibrium. We have,

- Case $n=1$ : The equation (13) $)_{3}$ at equilibrium, thanks to Equation (14), becomes $2 \frac{\partial^{2} \Delta H^{1}}{\partial \mu \partial \mu_{k i}} \lambda=0$ from which we have that $\frac{\partial \Delta H^{1}}{\partial \mu}$ can depend only on $\mu, \mu_{i}, \lambda, \lambda_{c}$; but the representation theorems show that no scalar function of order one with respect to equilibrium can depend only on these variables. It follows that $\frac{\partial \Delta H^{1}}{\partial \mu}=0$, so that $\Delta H^{1}$ is of degree zero with respect to $\mu$, and the property is verified for this case.

- Case $n \geq 2$ : Let us suppose, for the iterative hypothesis that $\Delta H$ up to order $n \geq 1$ with respect to equilibrium is a polynomial of degree $n-1$ in the variable $\mu$; we proceed now to prove that this property holds also with $n+1$ instead of $n$.

In fact, Equation $(13)_{1}$ up to order $n-1$ gives $\frac{\partial^{2} \Delta H^{n}}{\partial \mu \partial \mu_{i j}}=\frac{\partial^{2} \Delta H^{n+1}}{\partial \mu_{i} \partial \mu_{j}}$ from which we have:

$$
\Delta H^{n+1}=P_{n-2}+\Delta H_{i}^{n+1}\left(\mu, \mu_{a b}, \lambda, \lambda_{c}\right) \mu^{i}+\Delta H_{0}^{n+1}\left(\mu, \mu_{a b}, \lambda, \lambda_{c}\right)
$$

where $P_{n-2}$ is a polynomial of degree $n-2$ in $\mu$ and which is at least quadratic in $\mu_{j}$.

After that, Equation $(13)_{2}$ up to order $n$ gives $\frac{\partial^{2} \Delta H^{n+1}}{\partial \mu \partial \lambda_{i}}=\frac{\partial^{2} \Delta H^{n+1}}{\partial \lambda \partial \mu_{i}}$, which, thanks to Equation (58), becomes:

$$
\frac{\partial^{2} P_{n-2}}{\partial \mu \partial \lambda_{i}}+\frac{\partial^{2} \Delta H_{j}^{n+1}}{\partial \mu \partial \lambda_{i}} \mu_{j}+\frac{\partial^{2} \Delta H_{0}^{n+1}}{\partial \mu \partial \lambda_{i}}=\frac{\partial^{2} P_{n-2}}{\partial \lambda \partial \mu_{i}}+\frac{\partial \Delta H_{i}^{n+1}}{\partial \lambda}
$$


This relation, calculated in $\mu_{j}=0$, gives:

$$
\frac{\partial^{2} \Delta H_{0}^{n+1}}{\partial \mu \partial \lambda_{i}}=\frac{\partial \Delta H_{i}^{n+1}}{\partial \lambda}
$$

because $P_{n-2}$ is at least quadratic in $\mu_{j}$.

The derivative of Equation (59) with respect to $\mu_{j}$, calculated then in $\mu_{j}=0$, is $\frac{\partial^{2} \Delta H_{j}^{n+1}}{\partial \mu \partial \lambda_{i}}=$ $\left(\frac{\partial^{3} P_{n-2}}{\partial \mu_{j} \partial \lambda \partial \mu_{i}}\right)_{\mu_{j}=0}$ from which $\frac{\partial \Delta H_{j}^{n+1}}{\partial \lambda_{i}}=P_{n-1}^{i j}$ with $P_{n-1}^{i j}$ a polynomial of degree $n-1$ in $\mu$. By integrating this relation, we obtain $\Delta H_{i}^{n+1}=P_{n-1}^{i}+f_{n-1}^{i}\left(\mu, \mu_{a b}, \lambda\right.$, ) where $P_{n-1}^{i}$ is a polynomial of degree $n-1$ in $\mu$. However, for the representation theorems, a vectorial function, such as $f_{n-1}^{i}$, is zero, because it depends only on scalars and on a second order tensor. It follows that:

$$
\Delta H_{i}^{n+1}=P_{n-1}^{i}
$$

By using this result, Equation (60) can be integrated and gives:

$$
\frac{\partial \Delta H_{0}^{n+1}}{\partial \lambda_{i}}=P_{n}^{i}
$$

with $P_{n}^{i}$ a polynomial of degree $n$ in $\mu$.

Now, we impose Equation $(13)_{3}$ at order $n$ and see that its first, second, fifth and sixth terms are of degree $n-2$ in $\mu$, so that we have

$$
2 \frac{\partial^{2} \Delta H^{n+1}}{\partial \mu \partial \mu_{k i}} \lambda+2 \frac{\partial^{2} \Delta H^{n+1}}{\partial \mu_{k} \partial \mu_{i j}} \lambda_{j}=Q_{n-2}
$$

with $Q_{n-2}$ a polynomial of degree $n-2$ in $\mu$. This relation, thanks to Equation (58), becomes

$$
2 \lambda \frac{\partial^{2} \Delta H_{a}^{n+1}}{\partial \mu \partial \mu_{k i}} \mu^{a}+2 \lambda \frac{\partial^{2} \Delta H_{0}^{n+1}}{\partial \mu \partial \mu_{k i}}+2 \lambda_{j} \frac{\partial^{2} P_{n-2}}{\partial \mu_{k} \partial \mu_{i j}}+2 \lambda_{j} \frac{\partial}{\partial \mu_{i j}} \Delta H_{k}^{n+1}=Z_{n-2}
$$

with $Z_{n-2}$ a polynomial of degree $n-2$ in $\mu$. This relation, calculated in $\mu_{j}=0$, thanks to Equation (61) and to the fact that $P_{n-2}$ is at least quadratic in $\mu_{j}$, gives

$$
2 \lambda \frac{\partial^{2} \Delta H_{0}^{n+1}}{\partial \mu \partial \mu_{k i}}=\bar{Q}_{n-1}^{k i}
$$

with $\bar{Q}_{n-1}^{k i}$ a polynomial of degree $n-1$ in $\mu$. It follows that

$$
\frac{\partial \Delta H_{0}^{n+1}}{\partial \mu_{k i}}=\bar{P}_{n}^{k i}
$$

with $\bar{P}_{n}^{k i}$ a polynomial of degree $n$ in $\mu$. This result, jointly with Equation (62), gives that:

$$
\Delta H_{0}^{n+1}=\tilde{P}_{n}+f(\mu, \lambda) .
$$

However, a function depending only on $\mu$ and $\lambda$ cannot be of order $n+1$ with respect to equilibrium; it follows that $f(\mu, \lambda)=0$.

Consequently, Equations (58), (61) and (63) give that $\Delta H^{n+1}$ is a polynomial of degree $n$ in $\mu$, and this completes the proof. 
A further integration is possible for one combination of Equation (44) of the paper [24].

In fact, the integrability condition on $(44)_{1,4}$ of that paper allows us to obtain:

$$
\frac{\partial}{\partial \rho} h_{4}=-2 T^{2} \frac{\partial \varepsilon}{\partial T} \frac{\partial p}{\partial \rho}-2 \frac{T^{3}}{\rho^{2}}\left(\frac{\partial p}{\partial T}\right)^{2}=-2 \frac{\partial \varepsilon}{\partial T}\left(2 \frac{T}{\rho} h_{2}+\frac{5 T^{2} p}{3 \rho}\right) .
$$

(Here, and in the sequel, we use the notation of [24]. For example, the scalars $\beta_{2}, \beta_{3}$ are different from the constants with the same name of the present paper).

After that, by using $(44)_{2,3}$ and the present Equation (64), we obtain:

$$
\frac{\partial}{\partial \rho}\left[\beta_{2}-\frac{5}{6} \beta_{3}-\left(4 h_{2}+\frac{10}{3} p T\right)\left(\varepsilon+\frac{p}{\rho}\right)\right]=0 .
$$

Consequently, $\beta_{2}-\frac{5}{6} \beta_{3}-\left(4 h_{2}+\frac{10}{3} p T\right)\left(\varepsilon+\frac{p}{\rho}\right)$ may depend only on temperature.

Similarly, from $(44)_{5,6}$ and the present Equation (64), we obtain:

$$
\frac{\partial}{\partial T}\left[\beta_{2}-\frac{5}{6} \beta_{3}-\left(4 h_{2}+\frac{10}{3} p T\right)\left(\varepsilon+\frac{p}{\rho}\right)\right]=-\frac{1}{T}\left[\beta_{2}-\frac{5}{6} \beta_{3}-\left(4 h_{2}+\frac{10}{3} p T\right)\left(\varepsilon+\frac{p}{\rho}\right)\right],
$$

which is a differential equation for the unknown $\beta_{2}-\frac{5}{6} \beta_{3}-\left(4 h_{2}+\frac{10}{3} p T\right)\left(\varepsilon+\frac{p}{\rho}\right)$, whose solution is:

$$
\beta_{2}-\frac{5}{6} \beta_{3}-\left(4 h_{2}+\frac{10}{3} p T\right)\left(\varepsilon+\frac{p}{\rho}\right)=\frac{\text { constant }}{T} .
$$

However, in [26], it has been shown that $M_{i j k}$ is symmetric if and only if $L=\frac{5}{6} K$, as can be seen also from $(47)_{1,2}$ of [24]. This equation, for (48) and $(43)_{2}$ of [24], means that the left-hand side of the present Equation (67) is zero. Consequently, we have the symmetry of $M_{i j k}$ at first order, if and only if the constant arising from integration on the right-hand side of the present Equation (67) is zero! On the other hand, $m_{\text {pik }}$ at first order is already symmetric; eventually, its skew-symmetric parts may appear at higher orders with respect to equilibrium. This result is in agreement also with Chapter 5 of the book [38], where the symmetricity of $F^{i j k}$ and $G^{i k}$ at first order has been discussed.

\section{References}

1. Müller, I. Zum Paradoxen der Wärmeleitunstheorie. Z. Phys. 1967, 198, 329-344.

2. Ruggeri, T. Symmetric Hyperbolic System of Conservative Equations for a Viscous Conducting Fluid. Acta Mech. 1983, 47, 167-183.

3. Liu, I.-S.; Müller, I. Extended Thermodynamics of Classical and Degenerate Ideal Gases. Arch. Rat. Mech. Anal. 1983, 83, 285-332.

4. Liu, I.S.; Müller, I.; Ruggeri, T. Relativistic thermodynamics of gases. Ann. Phys. (N.Y.) 1986, 169, 191-219.

5. Müller, I.; Ruggeri, T. Rational Extended Thermodynamics, 2nd ed.; Springer: New York, NY, USA, 1998.

6. Müller, I. Entropy and Energy, —a Universal Competition. Entropy 2008, 10, 462-476.

7. Trovato, M.; Reggiani, L. Quantum maximum-entropy principle for closed quantum hydrodynamic transport within a Wigner function formalism. Phys. Rev. E 2011, 84, 061147. 
8. Trovato, M.; Reggiani, L. Quantum Maximum Entropy Principle for Fractional Exclusion Statistics. Phys. Rev. Lett. 2013, 110, 020404.

9. Carrisi, M.C.; Pennisi, S.; Ruggeri, T. The Lagrangian view-point compared with the Eulerian one, in the framework of Extended Thermodynamics. Acta Appl. Math. 2014, 132, 199-212.

10. Carrisi, M.C.; Pennisi, S. Waves speeds in the macroscopic relativistic extended model with many moments. Meccanica 2014, 49, 1493-1506.

11. Carrisi, M.C.; Pennisi, S. Extended Thermodynamics of Charged Gases with Many Moments: An Alternative Closure. J. Math. Phys. 2013, 54, 09301.

12. Carrisi, M.C.; Montisci, S.; Pennisi, S. Entropy Principle and Galilean Relativity for Dense Gases, the General Solution without Approximations. Entropy 2013, 15, 1035-1056.

13. Carrisi, M.C.; Pennisi, S. Extended Thermodynamics of charged gases with many moments. J. Math. Phys. 2013, 54, 023101.

14. Carrisi, M.C.; Pennisi, S. Some open problems in non-linear extended thermodynamics and their possible solutions. Ric. Mat. 2012, 60, 45-56.

15. Carrisi, M.C.; Farci, A.; Obounou, M.; Pennisi, S. Relativistic Extended Thermodynamics from the Lagrangian view-point. Ric. Mat. 2015, doi:10.1007/s11587-015-0244-x.

16. Ruggeri, T.; Trovato, M. Hyperbolicity in extended thermodynamics of Fermi and Bose gases. Continuum Mech. Thermodyn. 2004, 16, 551-576.

17. Barbera, E.; Brini, F. On stationary heat conduction in 3D symmetric domains: An application of extended thermodynamics. Acta Mech. 2010, 215, 241-260.

18. Barbera, E.; Brini, F.; Valenti, G. Some non-linear effects of stationary heat conduction in 3D domains through extended thermodynamics. Europhys. Lett. 2012, 98, 54004.

19. Barbera, E.; Brini, F. Heat transfer in gas mixtures: Advantages of an extended thermodynamics approach. Phys. Lett. A 2011, 375, 827-831.

20. Barbera, E.; Brini, F. Heat transfer in a binary gas mixture between two parallel plates: An application of linear extended thermodynamics. Acta Mech. 2011, 220, 87-105.

21. Barbera, E.; Brini, F. Heat transfer in multi-component gas mixtures described by extended thermodynamics. Meccanica 2012, 47, 655-666.

22. Barbera, E.; Brini, F. An extended thermodynamics description of stationary heat transfer in binary gas mixtures confined in radial symmetric bounded domains. Contin. Mech. Thermodyn. 2012, 24, 313-333.

23. Montisci, S.; Pennisi, S. Some Useful Tensorial Identies for Extended Thermodynamics. IEJPAM 2013, 6, 167-215.

24. Arima, T.; Taniguchi, S.; Ruggeri, T.; Sugiyama, M. Extended Thermodynamics of dense gases. Continuum Mech. Thermodyn. 2012, 24, 271-292.

25. Arima, T.; Taniguchi, S.; Ruggeri, T.; Sugiyama, M. Extended Thermodynamics of real gases with dynamic pressure: An extension of Meixner's theory. Phys. Lett. A 2012, 376, 2799-2803.

26. Arima, T.; Sugiyama, M. Characteristic features of Extended Thermodynamics of dense gases. Atti Accad. Pelorit. Pericol. 2013, 91 (Suppl. 1), doi:10.1478/AAPP.91S1A1.

27. Arima, T.; Taniguchi, S.; Ruggeri, T.; Sugiyama, M. Monoatomic rarefied gas as a singular limit of polyatomic gas in extended thermodynamics. Phys. Lett. A 2013, 377, 2136-2140. 
28. Pavic, M.; Ruggeri, T.; Simic, S. Maximum entropy principle for rarefied polyatomic gases. Phys. A 2013, 392, 1302-1317.

29. Arima, T.; Ruggeri, T.; Sugiyama, M.; Taniguchi, S. On the six-field model of fluids based on extended thermodynamics. Meccanica 2014, 49, 2181-2187.

30. Taniguchi, S.; Arima, T.; Ruggeri, T.; Sugiyama, M. Effect of dynamic pressure on the shock wave structure in a rarefied polyatomic gas. Phys. Fluids 2014, 26, 016103.

31. Arima, T.; Taniguchi, S.; Ruggeri, T.; Sugiyama, M. Dispersion relation for sound in rarefied polyatomic gases based on extended thermodynamics. Continuum Mech. Thermodyn. 2013, 25, 727-737.

32. Arima, T.; Mentrelli, A.; Ruggeri, T. Molecular Extended Thermodynamics of rarefied polyatomic gases and wave velocities for increasing number of moments. Ann. Phys. 2014, 345, 111-132.

33. Carrisi, M.C.; Pennisi, S. An 18 Moments Model for Dense Gases: Entropy and Galilean Relativity Principles without Expansions. Entropy 2015, 17, 214-230.

34. Taniguchi, S.; Arima, T.; Ruggeri, T.; Sugiyama, M. Thermodynamic theory of the shock wave structure in a rarefied polyatomic gas: Beyond the Bethe-Teller theory. Phys. Rev. E 2014, 89, 013025.

35. Arima, T.; Barbera, E.; Brini, F.; Sugiyama, M. The role of the dynamic pressure in stationary heat conduction of a rarefied polyatomic gas. Phys. Lett. A 2014, 378, 2695-2700.

36. Barbera, E.; Brini, F.; Sugiyama, M. Heat Transfer Problem in a Van Der Waals Gas. Acta Appl. Math. 2014, 132, 41-50.

37. Carrisi, C.; Pennisi, S. Extended thermodynamics for dense gases up to whatever order. Int. J. Non-Linear Mech. 2015, 77, 74-84.

38. Ruggeri, T.; Sugiyama, M. Rational Extended Thermodynamics beyond the Monatomic Gas; Springer: Berlin/Heidelberg, Germany, 2015.

39. Liu, I.-S. Method of Lagrange Multipliers for Exploitation of the Entropy Principle. Arch. Rat. Mech. Anal. 1972, 46, 131-148.

40. Ruggeri, T.; Strumia, A. Main Field and Convex Covariant Density for Quasi-Linear Hyperbolic Systems. Relativistic Fluid Dynamics. Ann. Inst. H. Poincaré. 1981, 34, 65-84.

41. Ruggeri, T. Galilean Invariance and Entropy Principle for Systems of Balance Laws. The Structure of the Extended Thermodynamics. Continuum. Mech. Thermodyn. 1989, 1, 3-20.

42. Pennisi, S.; Ruggeri, T. A new method to exploit the Entropy Principle and Galilean Invariance in the macroscopic approach of Extended Thermodynamics. Ric. Mat. 2006, 55, 319-339.

43. Pennisi, S.; Trovato, M. On the Irreducibility of Professor G.F. Smith's Representations for isotropic functions. Int. J. Engng Sci. 1987, 25, 1059-1065.

44. Carrisi, M.C.; Montisci, S.; Pennisi, S. Representation Theorems In A 4-Dimensional Euclidean Space. The Case With Only Skew-Symmetric Tensors. Int. J. Pure Appl. Math. 2014, 93, 929-979.

(c) 2015 by the authors; licensee MDPI, Basel, Switzerland. This article is an open access article distributed under the terms and conditions of the Creative Commons Attribution license (http://creativecommons.org/licenses/by/4.0/). 